\title{
Seismic performance of buildings retrofitted with nonlinear viscous dampers and adjacent reaction towers
}

\author{
Nicola Impollonia ${ }^{1}$, Alessandro Palmeri ${ }^{2 * \dagger}$ \\ ${ }^{1}$ Department of Civil Engineering and Architecture, University of Catania, \\ Viale Andrea Doria 6, 95123, Catania, Italy \\ 2 School of Architecture, Building and Civil Engineering, Sir Frank Gibb Building, Loughborough University, \\ Loughborough, LE11 3RN, United Kingdom
}

\begin{abstract}
SUMMARY
An effective strategy of seismic retrofitting consists of installing nonlinear viscous dampers (NLVDs) between the existing building, with insufficient lateral resistance, and some auxiliary towers, specially designed and erected as reaction structures. This allows improving the seismic performance of the existing building without any major alteration to its structural and nonstructural elements, which makes this approach particularly appealing for buildings with heritage value. In this paper, the nonlinear governing equations of the coupled lateral-torsional seismic motion are derived from first principles for the general case of a multi-story building connected at various locations in plan and in elevation to an arbitrary number of multi-story towers. This formulation is then used to assess the performance of the proposed retrofitting strategy for a real case study, namely a five-story student hall of residence in the city of Messina, Italy. The results of extensive time-history analyses highlight the key design considerations associated with the stiffness of the reaction towers and the mechanical parameters of the NLVDs, confirming the validity of this approach. Copyright (c) 2017 John Wiley \& Sons, Ltd.
\end{abstract}

Received ...

KEY WORDS: Coupled adjacent buildings; Optimal passive control; Nonlinear energy dissipation devices; Seismic retrofitting; Time-history dynamic analyses; Viscous dampers.

\section{INTRODUCTION}

Improving the seismic performance of existing buildings is often a challenging task for a structural engineer. Such a circumstance may arise, for instance, when a higher importance class is required for the building, or when the map of the seismic hazard is updated with higher values of the spectral acceleration for a given return period. As an example, the new Italian seismic code [1] has substantially increased the seismic hazard level across the country, meaning that the safety of an enormous stock of existing buildings needed to be checked against the increased seismic demand, and some form of structural retrofitting is very often required.

In this respect, two major strategies may be considered: $i$ ) strengthening, i.e. increasing the capacity of the structural members; and/or $i i$ ) reducing the seismic demand, e.g. with the installation of seismic protective devices, such as dampers and isolators (see for instance Refs. [2-8]). In certain cases, the former strategy could be more convenient for relatively small-sized buildings, whereas the latter tends to be more advantageous for large structures, including key infrastructural facilities (e.g. schools, university buildings, hospitals, etcetera), so to keep to a minimum any service interruption (although seismic dampers can be effective and cost-efficient also for retrofitting low-rise buildings, see e.g. Ref. [9]). Financing considerations may also discourage the strengthening option for relatively large structures, as an accurate estimate of the costs may be very difficult, and the project can easily exceed the budget initially set up for

${ }^{*}$ Correspondence to: Dr. Alessandro Palmeri, School of Civil and Building Engineering, Sir Frank Gibb Building, Loughborough University, Loughborough, LE11 3RN, United Kingdom

†E-mail: A.Palmeri@Lboro.ac.uk, Dynamics.Structures@Gmail.com 
the intervention. Furthermore, this type of retrofitting is usually spread out over the whole structure, and may interfere with many non-structural components, while the installation of seismic isolators and dampers can often be optimized to limit such issues. On the other hand, the latter approach can be quite problematic in the case of buildings of special architectural or historic interest, as the competent authorities may require the maximum degree of preservation, which is often incompatible with the work required to fit in the seismic devices.

The case-study building considered in this paper poses such a challenge. Erected in the 1930s in the heart of the city of Messina (Italy), this five-story building has been used since then as a university hall of residence. As the seismic demand dictated by the new Italian code exceeds the earthquake resistance of the structural frame, made of brick masonry confined by reinforced concrete $(\mathrm{RC})$ members, a retrofitting intervention is needed to keep the building in use. Considering its historic value, the installation of dissipative devices mounted on $\mathrm{X}$ or chevron steel braces would be overly invasive and therefore unacceptable; the installation of seismic isolators would require extensive foundation works, making this option too expensive and technically challenging; on the other hand, the very low level of ductility of the existing structural system means that an adequate strengthening of beams and column could be extremely costly. As these three approaches are not fully satisfactory, an alternative strategy of seismic retrofitting is considered in the following, in which nonlinear viscous dampers (NLVDs) are installed between the existing structure and three adjacent towers, specially designed to work as reaction structures, as preliminary investigated by Impollonia et al. [10]. Advantages of this solution include: $i$ ) the NLVDs act in the horizontal plane, which allows maximizing their effects; $i i)$ it is possible to avoid the impact of the damping forces on the existing foundation, which is usually difficult and expensive to retrofit. However, the main constraint exists that, like in the case-study building, space must be available for the erection of the reaction towers. Furthermore, the existing structure may require local strengthening, or the addition of steel spreading elements, to cope with the large concentrated forces applied by the dampers.

The idea of using viscous-fluid dampers connected to adjacent structures is not new, although previous work has been mainly focussed on new buildings and linear dampers. Among others, Xu et al. [11] have demonstrated that the elastic stiffness in the classical Kelvin-Voigt model of linear devices does not play an important role in the seismic response of the building, unless it becomes very high, which inevitably reduces their efficiency. The linear KelvinVoigt model has also been adopted by Zhang and Xu [12] for identifying the optimal parameters of the dampers which maximize either the modal viscous damping ratios or the dynamic response to a stationary Kanai-Tajimi filtered white noise. Zhang and $\mathrm{Xu}$ [13] have shown that, for applications to adjacent buildings with the same height and mass at each floor, the analyses with Maxwell and Kelvin-Voigt models (with the elastic stiffness either in series or in parallel) provide similar results. Aida et al. [14] have derived an approximate tuning method for linear structures interconnected by single linear Kelvin-Voigt damper.

$\mathrm{Ni}$ et al. [15] have used the stochastic linearization technique to evaluate the random seismic response of linear structures interconnected by an arbitrary number of nonlinear hysteretic dampers, represented with the classical BoucWen differential model, also considering different soil condition with a stationary Kanai-Tajimi filtered white noise. The results of their parametric study demonstrate that nonlinear dampers have the potential to provide a wideband mitigation of earthquake-induced vibrations in both low- and high-frequency range.

Patel and Jangid [16] have pointed out that lesser dampers at appropriate locations can reduce the seismic response of the connected structures almost as much as when they are connected at all floors, which provides opportunities for reducing the cost of the retrofitting intervention. Such a result has also been confirmed by experimental testing on two building models of 1:4 length scale, connected by linear fluid dampers, carried out by a seismic simulator [17]. Bigdeli et al. [18] have investigated five alternative approaches to identify the optimal arrangement of a limited number of dampers which minimize the inter-story drifts, showing that increasing the number of devices does not necessarily improve the efficiency of the intervention.

Trombetti and Silvestri [19], within the class of Rayleigh-type linear damping, have analyzed the performance of stiffness-proportional and mass-proportional dampers: while the former case represents the traditional installation of energy dissipation devices (EDDs) connecting adjacent stories, in the latter case the dampers are ideally connected to a rigid lateral-resisting element. Their parametric study shows that mass-proportional arrangement provides the largest damping effect, providing a theoretical justification for the use of dampers connected to adjacent structures.

Lavan [20] has used an analytical formulation with non-dimensional quantities to assess the efficiency of linear viscus dampers in wall-EDD-wall systems, showing that the main effect of the additional damping is to reduce the pseudo-accelerations in the structure. Lavan and Abecassis [21] have studied wall-EDD-frame systems, showing with an extensive parametric analysis that the seismic response of an existing frame can be efficiently reduced by connecting it to new walls with linear viscous dampers. 
In terms of comparison between linear and nonlinear devices, Martinez-Rodrigo and Romero [22] have considered the case of viscous dampers mounted on chevron-braced frames with different values of the velocity exponent, revealing that a more pronounced reduction of the peak accelerations is achieved with the linear dampers, while increasing the nonlinearity results in smaller forces in the dampers. A similar trend of results has been identified by Dall'Asta et al. [23] using a probabilistic performance-based approach; the latter study has also shown that the nonlinear behavior of the viscous dampers allows reducing the variability in the reaction forces in both the dampers and the lateral-resisting frame. Zhu et al. [24] and Tubaldi [25], amongst others, have used linear dynamic models to identify the optimal values of viscous and viscoelastic dampers connecting two adjacent building structures. Probabilistic performance-based procedures have been proposed in Refs. [26] and [27] to assess the seismic risk associated with the seismic pounding between adjacent buildings and to quantify the risk reduction achieved with the use of viscous and viscoelastic dampers. Yang and Lam [28] have demonstrated that torsional effects on two adjacent asymmetrical building may be adversely affected by interconnected dampers, meaning that mass eccentricities must be taken into account when selecting the dampers.

Roia et al. [29] have reported the results of ambient vibrations tests performed before and after the seismic retrofitting of an RC frame building through two external steel truss towers, each one rigidly connected with the top floors of the existing structure and equipped with viscous dampers. Their investigations confirm an increase in the lateral stiffness of the building due to its coupling with the auxiliary towers, while the very low amplitude of the ambient vibration did not show any significant effect of the dissipative devices in terms of additional damping.

Passoni et al. [30] have presented a review of previous studies on the coupling of buildings structures with dissipative connections, highlighting that both displacement- and velocity-activated dampers can be successfully used to improve the seismic performance of an existing structure.

In this paper, the equations of motion governing the seismic response of a primary multi-story building connected to an arbitrary number of secondary reaction towers through NLVDs have been rigorously derived from first principles and numerically integrated. The devised three-dimensional mathematical model has then been used to investigate the effects of the key design parameters, namely $i$ ) the relative stiffness of the towers, $i i$ ) the mechanical parameters of the dampers (velocity exponent and viscous coefficient) and $i i i$ ) the number and height of the connected stories, as well as $i v$ ) the effects of the intensity of the seismic event and $v$ ) the ability of the retrofitting strategy to control coupled lateral-torsional vibrations.

All the analyses have been carried out on the selected case-study structure, deemed as representative of a large stock of edifices with historical significance that worldwide need seismic improvements without compromising their architectural features and the existing functionalities. The results of an extensive parametric campaign of nonlinear time-history analyses (in excess of 60,000) have been scrutinized transcending the actual case study, which has then allowed to highlight the main trends in the seismic response and to elucidate for the benefit of the structural designers which considerations are important when approaching this type of seismic retrofitting and how its performance can be maximized.

\section{FORMULATION}

Aim of this section is to present the computational model used for the nonlinear seismic analysis of the combined building-damper-tower system. The governing equations of motion have been derived from first principles, which provide more insight into the problem with respect to the use of commercial finite element analysis software. The generality of the model has been guaranteed by considering an arbitrary number of stories, dampers and towers, as well as an arbitrary relative location of the individual structures with respect to each other and arbitrary position and orientation of the building-tower dissipative links. This has allowed a variety of parametric investigations to be carried out (including a complex-valued modal analysis for the case of linear dampers), which would have been much more cumbersome with a commercial software.

\subsection{Individual sub-frames}

Without lack of generality, a three-dimensional shear-type model is assumed for both the main building to be retrofitted (denoted as sub-frame $j=0$ ) and the $n_{\mathrm{R}}$ reaction towers (sub-frames $j>0$, with $j=1, \cdots, n_{\mathrm{R}}$ ). All sub-frames have:

- the same number of stories $n_{\mathrm{S}}$ and the same inter-story heights, so the seismic dampers can be installed horizontally; 


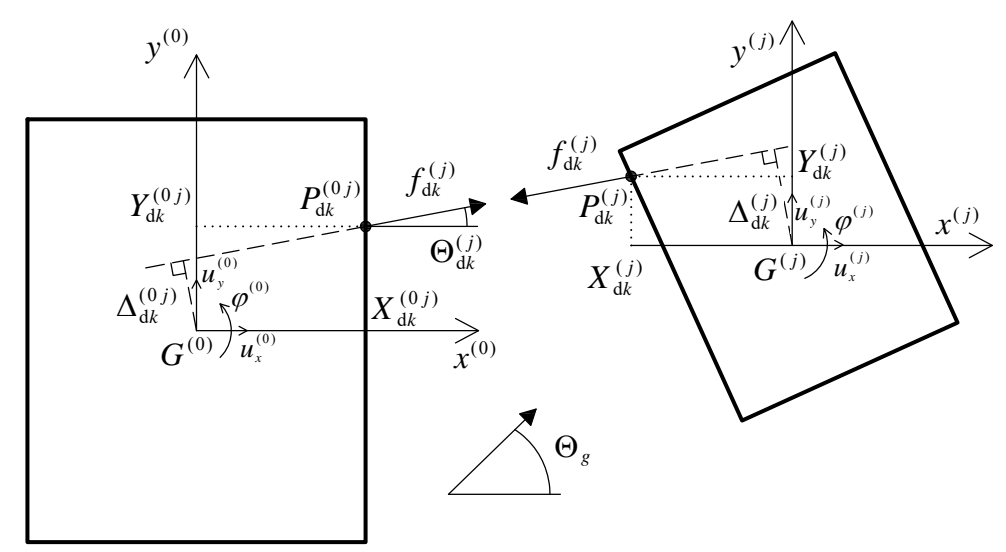

Figure 1. Plane view of the $i$ th floor showing the $j$ th reaction tower connected to the main building

- masses concentrated at the story levels, with slabs perfectly rigid in their own plane (diaphragmatic constraint), meaning that the degrees of freedom (DoFs) reduce to three per each story, i.e. the $x$ and $y$ horizontal translations of the centroid $\mathrm{G}_{i}^{(j)}$ at the $i$ th level of the $j$ th sub-frame, say $u_{x i}^{(j)}(t)$ and $u_{y i}^{(j)}(t)$, and the rotation $\varphi_{i}^{(j)}(t)$ about the vertical axis, as shown in Figure 1;

- linear-elastic behavior, as it is anticipated that, in order to maximize the benefits of NLVDs, no plastic hinge will form in the existing building and in any of the reaction towers;

- elastic stiffness provided by shear walls and columns at each level, while the beams are assumed to be perfectly rigid (shear-type model);

- classical damping, i.e. damping proportional to mass and stiffness (when individually considered).

Accordingly, the seismic vibration of the $j$ th sub-frame is ruled by:

$$
\mathbf{m}^{(j)} \cdot \ddot{\mathbf{u}}^{(j)}(t)+\mathbf{c}^{(j)} \cdot \dot{\mathbf{u}}^{(j)}(t)+\mathbf{k}^{(j)} \cdot \mathbf{u}^{(j)}(t)+\gamma_{\mathrm{d}}^{(j)} \cdot \mathbf{f}_{\mathrm{d}}^{(j)}(t)=-\mathbf{m}^{(j)} \cdot \boldsymbol{\tau} \cdot \ddot{\mathbf{u}}_{\mathrm{g}}(t),
$$

where $\mathbf{m}^{(j)}, \mathbf{c}^{(j)}$ and $\mathbf{k}^{(j)}$ are the $\left(3 n_{\mathrm{S}} \times 3 n_{\mathrm{S}}\right)$ matrices of mass, equivalent (linear) viscous damping and elastic stiffness of the $j$ th sub-frame, individually considered; $\mathbf{u}^{(j)}(t)=\left\{\left\{\mathbf{u}_{x}^{(j)}(t)\right\}^{\top}\left\{\mathbf{u}_{y}^{(j)}(t)\right\}^{\top}\left\{\mathbf{u}_{\varphi}^{(j)}(t)\right\}^{\top}\right\}^{\top}$ is the array collecting its $3 n_{\mathrm{S}}$ DoFs, partitioned into the three blocks, namely: $\mathbf{u}_{x}^{(j)}(t)=\left\{u_{x 1}^{(j)}(t) \cdots u_{x n_{\mathrm{S}}}^{(j)}(t)\right\}^{\top}$, with the translations along $x ; \mathbf{u}_{y}^{(j)}(t)=\left\{u_{y 1}^{(j)}(t) \cdots u_{y n_{\mathrm{S}}}^{(j)}(t)\right\}^{\top}$, with the translations along $y$; and $\mathbf{u}_{\varphi}^{(j)}(t)=$ $\left\{\varphi_{1}^{(j)}(t) \cdots \varphi_{n \mathrm{~S}}^{(j)}(t)\right\}^{\top}$, with the rotations about the vertical axis. Furthermore: $\mathbf{f}_{\mathrm{d}}^{(j)}(t)=\left\{f_{\mathrm{d} 1}^{(j)}(t) \cdots f_{\mathrm{d} n_{\mathrm{D} j}}^{(j)}(t)\right\}^{\top}$ is the array listing the nonlinear damping forces exerted on the $j$ th sub-frame by the $n_{\mathrm{D} j}$ NLVDs connected to it (positive if they pull on the $j$ th sub-frame, meaning that tensile forces are applied at the ends of the NLVDs); $\gamma_{\mathrm{d}}^{(j)}$ is the associated $\left(3 n_{\mathrm{S}} \times n_{\mathrm{D} j}\right)$ influence matrix; $\ddot{\mathbf{u}}_{\mathrm{g}}(t)=\left\{\ddot{u}_{\mathrm{g} p}(t) \ddot{u}_{\mathrm{g} q}(t)\right\}^{\top}$ is the array with the two components of the ground acceleration along the orthogonal directions $p$ and $q ; \tau$ is the associated $\left(3 n_{\mathrm{S}} \times 2\right)$ incidence matrix; the over-dot $\left({ }^{\circ}\right)$ means derivation with respect to the time $t$, while the central dot $(\cdot)$ means matrix product and $\mathrm{T}$ is the transpose operator.

It is worth noting here that:

- the shear-type assumption for the stiffness of the sub-frames can be relaxed through the so-called static condensation of the rotational DoFs not associated with inertial forces (typically, beam-to-column relative rotations in the vertical plan) and does not affect the generality of the mathematical formulation;

- $\ddot{\mathbf{u}}_{\mathrm{g}}(t)$ and $\boldsymbol{\tau}$ are the same for all the sub-frames, i.e. any effect due to spatial variation of the ground motion and structure-soil-structure interaction is neglected, and the reference systems $\mathrm{G}_{x^{(j)} y^{(j)}}^{(j)}$ (with $\left.j=0, \cdots, n_{\mathrm{R}}\right)$ for all the sub-frames are parallel to each other;

- the total number of NLVDs is $n_{\mathrm{Dtot}}=n_{\mathrm{D} 0}=\sum_{j=1}^{n_{\mathrm{R}}} n_{\mathrm{D} j}$, as they connect the main building to the $n_{\mathrm{R}}$ reaction towers (while there is no connection between any two of them); 
- the $n_{\text {Dtot }}$-dimensional array $\mathbf{f}_{\mathrm{d}}^{(0)}(t)$ and the corresponding $\left(3 n_{\mathrm{S}} \times n_{\text {Dtot }}\right)$ matrix $\gamma_{\mathrm{d}}^{(0)}$ for the main building $(j=0)$ can be partitioned as follows:

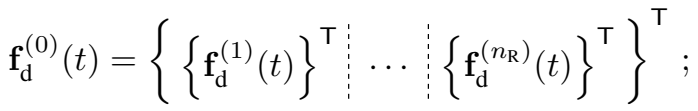

$$
\begin{aligned}
& \gamma_{\mathrm{d}}^{(0)}=\left[\begin{array}{l:l:l}
\gamma_{\mathrm{d}}^{(01)} & \cdots & \gamma_{\mathrm{d}}^{\left(0 n_{\mathrm{R}}\right)}
\end{array}\right],
\end{aligned}
$$

in which the generic block $\gamma_{\mathrm{d}}^{(0 j)}$ is the $\left(3 n_{\mathrm{S}} \times n_{\mathrm{D} j}\right)$ influence matrix on the main building for the array $\mathbf{f}_{\mathrm{d}}^{(j)}(t)$.

The matrices $\gamma_{\mathrm{d}}^{(j)}$ and $\gamma_{\mathrm{d}}^{(0 j)}$ can be easily built column by column. Indeed, if the $k$ th damper in the $j$ th reaction tower is located at the $i$ th story, then the only non-zero elements in the $k$ th columns $\gamma_{\mathrm{d} k}^{(j)}$ and $\gamma_{\mathrm{d} k}^{(0 j)}$ of the above matrices are those at the row positions $i, i+n_{\mathrm{S}}$ and $i+2 n_{\mathrm{S}}$; that is:

$$
\begin{gathered}
\left\{\gamma_{\mathrm{d} k}^{(j)}\right\}_{r}= \begin{cases}-\cos \left(\Theta_{\mathrm{d} k}^{(j)}\right), & \text { if } r=i ; \\
-\sin \left(\Theta_{\mathrm{d} k}^{(j)}\right), & \text { if } r=i+n_{\mathrm{S}} \\
\Delta_{\mathrm{d} k}^{(j)}=Y_{\mathrm{d} k}^{(j)} \cos \left(\Theta_{\mathrm{d} k}^{(j)}\right)-X_{\mathrm{d} k}^{(j)} \sin \left(\Theta_{\mathrm{d} k}^{(j)}\right), & \text { if } r=i+2 n_{\mathrm{S}} \\
0, & \text { otherwise }\end{cases} \\
\left\{\gamma_{\mathrm{d} k}^{(0 j)}\right\}_{r}= \begin{cases}\cos \left(\Theta_{\mathrm{d} k}^{(j)}\right), & \text { if } r=i \\
\sin \left(\Theta_{\mathrm{d} k}^{(j)}\right), & \text { if } r=i+n_{\mathrm{S}} \\
\Delta_{\mathrm{d} k}^{(0 j)}=X_{\mathrm{d} k}^{(0 j)} \sin \left(\Theta_{\mathrm{d} k}^{(j)}\right)-Y_{\mathrm{d} k}^{(0 j)} \cos \left(\Theta_{\mathrm{d} k}^{(j)}\right), & \text { if } r=i+2 n_{\mathrm{S}} \\
0, & \text { otherwise }\end{cases}
\end{gathered}
$$

where (see Figure 1): $\Theta_{\mathrm{d} k}^{(j)}$ is the angle that the damper forms with the $x$ axis, positive if anti-clockwise; $\left\{X_{\mathrm{d} k}^{(0 j)}, Y_{\mathrm{d} k}^{(0 j)}\right\}$ and $\left\{X_{\mathrm{d} k}^{(j)}, Y_{\mathrm{d} k}^{(j)}\right\}$ are the coordinates in plane of the points $\mathrm{P}_{\mathrm{d} k}^{(0 j)}$ and $\mathrm{P}_{\mathrm{d} k}^{(j)}$ representing the damper's ends in the reference systems $\mathrm{G}_{x^{(0)} y^{(0)}}^{(0)}$ and $\mathrm{G}_{x^{(j)} y^{(j)}}^{(j)}$ of main building and $j$ th reaction tower, respectively; and $\Delta_{\mathrm{d} k}^{(0 j)}$ and $\Delta_{\mathrm{d} k}^{(j)}$ are the lever arms of the damper force $f_{\mathrm{d} k}^{(j)}$ in the 0 th and $j$ th sub-frame (positive if the pulling force $f_{\mathrm{d} k}^{(j)}>0$ results into an anticlockwise torque on the sub-frames).

The matrices $\mathbf{m}^{(j)}, \mathbf{k}^{(j)}$ and $\boldsymbol{\tau}$ can be assembled as:

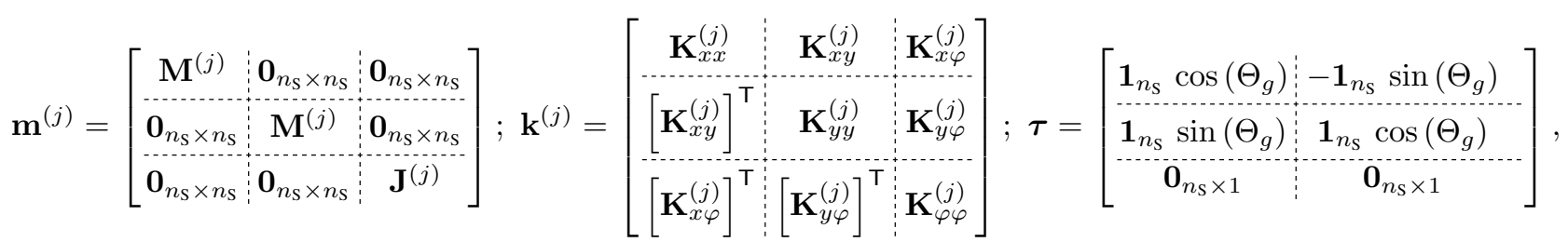

where: $\mathbf{M}^{(j)}=\operatorname{diag}\left\{M_{1}^{(j)}, \cdots, M_{n_{\mathrm{S}}}^{(j)}\right\}$ and $\mathbf{J}^{(j)}=\operatorname{diag}\left\{J_{1}^{(j)}, \cdots, J_{n_{\mathrm{S}}}^{(j)}\right\}$ are two diagonal matrices listing masses and polar moments of the $n_{\mathrm{S}}$ stories for the $j$ th sub-frame; $\mathbf{K}_{r s}^{(j)}$ is the $\left(n_{\mathrm{S}} \times n_{\mathrm{S}}\right)$ block of stiffness matrix associated with the DoFs of the $j$ th sub-frame in the $r$ th and $s$ th directions $(r=x, y, \varphi ; s=x, y, \varphi) ; \mathbf{0}_{r \times s}$ denotes a zero matrix with $r$ rows and $s$ columns and $\mathbf{1}_{s}$ is a unit vector of size $s ; \Theta_{g}$ is the angle that the component $p$ of the accelerogram forms with the $x$ axis (see Figure 1). If all the lateral resisting elements (shear walls and columns) are parallel to $x$ and $y$, then the block $\mathbf{K}_{x y}^{(j)}$ reduces to zero; if the center of mass $G_{j}^{(j)}$ coincides at all stories with the center of rigidity, then the blocks $\mathbf{K}_{x \varphi}^{(j)}$ and $\mathbf{K}_{y \varphi}^{(j)}$ become zero as well.

A reasonable assumption for the formation of the viscous damping matrix $\mathbf{c}^{(j)}$ is that the modes of vibration of the $j$ th sub-frame have the same viscous damping ratio $\zeta^{(j)}$; accordingly, the following expression can be used:

$$
\mathbf{c}^{(j)}=2 \zeta^{(j)} \mathbf{m}^{(j)} \cdot \boldsymbol{\Phi}^{(j)} \cdot \boldsymbol{\Omega}^{(j)} \cdot\left[\boldsymbol{\Phi}^{(j)}\right]^{\top} \cdot \mathbf{m}^{(j)}
$$


where $\boldsymbol{\Phi}^{(j)}=\left[\phi_{j 1} \cdots \phi_{j m_{j}}\right]$ and $\boldsymbol{\Omega}^{(j)}=\operatorname{diag}\left\{\omega_{j 1}, \cdots, \omega_{j m_{j}}\right\}$ are the $\left(3 n_{\mathrm{S}} \times m_{j}\right)$ modal matrix and the $\left(m_{j} \times m_{j}\right)$ spectral matrix of the $j$ th sub-frame, solution of the real-valued eigenproblem:

$$
\mathbf{k}^{(j)} \cdot \phi_{j \ell}=\omega_{j \ell}^{2} \mathbf{m}^{(j)} \cdot \boldsymbol{\phi}_{j \ell},
$$

in which the modal shapes satisfy the normalisation condition $\phi_{j \ell}^{\top} \cdot \mathbf{m}^{(j)} \cdot \phi_{j \ell}=1$ and the modal circular frequencies are ordered such that $\omega_{j \ell} \geq \omega_{j(\ell-1)}$, with $\ell \leq m_{j} \leq 3 n_{\mathrm{S}}, m_{j}$ being the number of modes computed for the $j$ th subframe. Importantly, in order to capture at least $90 \%$ of the inherent damping forces in the $j$ th sub-frame, the following inequalities shall be satisfied:

$$
\left\{\begin{array}{l}
\sum_{\ell=1}^{m_{j}}\left(\phi_{j \ell}^{\top} \cdot \mathbf{m}^{(j)} \cdot \boldsymbol{\tau}_{p}\right)^{2} \geq 0.90 M_{\mathrm{tot}}^{(j)} \\
\sum_{\ell=1}^{m_{j}}\left(\phi_{j \ell}^{\top} \cdot \mathbf{m}^{(j)} \cdot \boldsymbol{\tau}_{q}\right)^{2} \geq 0.90 M_{\mathrm{tot}}^{(j)}
\end{array}\right.
$$

where $\tau_{p}$ and $\tau_{q}$ are the first and the second column of the seismic incidence matrix $\boldsymbol{\tau}$ and $M_{\text {tot }}^{(j)}=\sum_{i=1}^{n_{\mathrm{s}}} M_{i}^{(j)}$ is the total mass of the $j$ th sub-frame. This representation of the damping matrix (see e.g. Refs. [31, 32] ) is preferable to the use of the Rayleigh damping, as it allows having the same value of viscous damping ratio for all the seismically significant modes of vibration of each individual sub-frame

\subsection{Nonlinear viscous dampers}

The primary source of energy dissipation in the proposed retrofitting approach comes from the NLVDs, which connect horizontally the main (existing) building to the (new) $n_{\mathrm{R}}$ reaction towers, making nonlinear and non-classical the damping of the resulting coupled dynamic system, which has in total $n_{\text {tot }}=3 n_{\mathrm{S}}\left(n_{\mathrm{R}}+1\right)$ DoFs. by:

The reaction force of the $k$ th NLVD installed between the main building and the $j$ th reaction tower, $f_{\mathrm{d} k}^{(j)}(t)$, is ruled

$$
f_{\mathrm{d} k}^{(j)}(t)=c_{\mathrm{d} k}^{(j)} G_{\mathrm{d}}\left\{v_{\mathrm{d} k}^{(j)}(t)\right\}
$$

where: $c_{\mathrm{d} k}^{(j)}$ is the damping constant (measured in $\left.\mathrm{kN}\right) ; v_{\mathrm{d} k}^{(j)}(t)$ is the relative velocity between the damper's ends along its axis (positive if the device is stretching, resulting in a positive pulling force); and $G_{\mathrm{d}}$ is a dimensionless nonlinear function, so defined:

$$
G_{\mathrm{d}}\{v\}=\operatorname{sgn}(v)\left|\frac{v}{v_{\mathrm{d} 0}}\right|^{\alpha_{\mathrm{d}}}
$$

in which the dimensionless exponent $\alpha_{\mathrm{d}}$ (with $0<\alpha_{\mathrm{d}} \leq 1$ ) controls the shape of the energy dissipation loops and $v_{\mathrm{d} 0}$ is the reference value of the velocity of the damper (typically assumed as $v_{\mathrm{d} 0}=1 \mathrm{~m} / \mathrm{s}$ ), introduced in order use the same dimensions for the damping constant $c_{\mathrm{d} k}^{(j)}$ irrespectively of the exponent $\alpha_{\mathrm{d}}$. The limiting case of a linear viscous damper is obtained with $\alpha_{\mathrm{d}}=1$, as the viscous force $f_{\mathrm{d} k}^{(j)}(t)$ then becomes proportional to the relative velocity $v_{\mathrm{d} k}^{(j)}(t)$. More generally, the lesser the exponent $\alpha_{\mathrm{d}}$, the higher the level of nonlinearity. In the following, the same value of the exponent $\alpha_{\mathrm{d}}$ will be used for all the dampers used in the retrofitting intervention, but this assumption can be easily relaxed if needed.

Introducing now the array $\mathbf{v}_{\mathrm{d}}^{(j)}(t)=\left\{v_{\mathrm{d} 1}^{(j)}(t) \cdots v_{\mathrm{d} n_{\mathrm{D} j}}^{(j)}(t)\right\}^{\top}$, which collects all the relative velocities at the ends of the $n_{\mathrm{D} j}$ NLVDs installed with the $j$ th reaction tower, Eq. (8) can be expanded as:

$$
\mathbf{f}_{\mathrm{d}}^{(j)}(t)=\mathbf{c}_{\mathrm{d}}^{(j)} G_{\mathrm{d}}\left\{\mathbf{v}_{\mathrm{d}}^{(j)}(t)\right\}
$$

in which: $\mathbf{c}_{\mathrm{d}}^{(j)}=\operatorname{diag}\left\{c_{\mathrm{d} 1}^{(j)} \cdots c_{\mathrm{d} n_{\mathrm{D} j}}^{(j)}\right\}$ is the diagonal matrix listing the $n_{\mathrm{D} j}$ damping constants; while the array of the relative velocities in the right-hand side can be expressed as a function of the translational and rotational velocities of the sub-frames 0 and $j$ :

$$
\mathbf{v}_{\mathrm{d}}^{(j)}(t)=\left[\gamma_{\mathrm{d}}^{(0 j)}\right]^{\top} \cdot \dot{\mathbf{u}}^{(0)}(t)+\left[\gamma_{\mathrm{d}}^{(j)}\right]^{\top} \cdot \dot{\mathbf{u}}^{(j)}(t)
$$




\subsection{Coupled dynamic system}

The equations of motion for the $n_{n_{\mathrm{R}}}+1$ sub-frames (Eq. (1)) along with the equations governing the dynamic response of the $n_{\text {Dtot }}$ NLVDs (Eqs. (2), (10) and (11)) lend themselves to be cast as a compact matrix equation of motion (similar to Eq. (1)):

$$
\mathbf{M} \cdot \ddot{\mathbf{U}}(t)+\mathbf{C} \cdot \dot{\mathbf{U}}(t)+\mathbf{K} \cdot \mathbf{U}(t)+\boldsymbol{\Gamma}_{\mathrm{d}} \cdot \mathbf{F}_{\mathrm{d}}(t)=-\mathbf{M} \cdot \mathbf{T} \cdot \ddot{\mathbf{u}}_{\mathrm{g}}(t),
$$

where: $\mathbf{M}, \mathbf{C}$ and $\mathbf{K}$ are the block-diagonal matrices of size $n_{\text {tot }}$ that collect the mass, damping and stiffness matrices of the individual sub-frames:

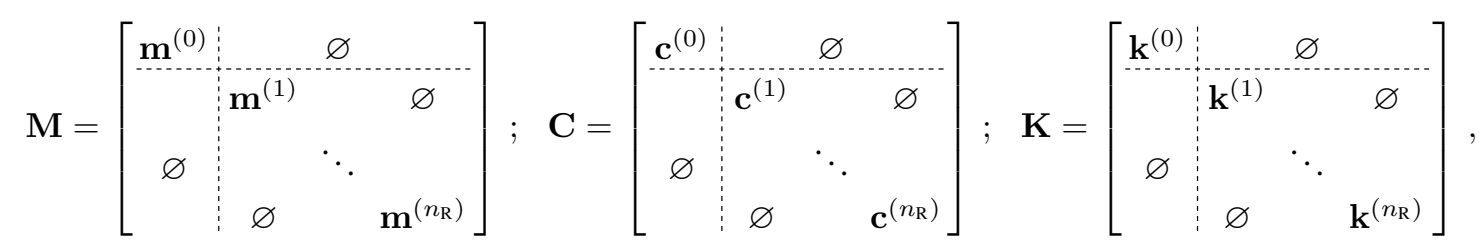

in which the symbol $\varnothing$ reminds that all the out-of-diagonal blocks are zero matrices; the tall rectangular matrix $\mathbf{T}=$ $\left[\begin{array}{l:lll}\boldsymbol{\tau}^{\top} & \boldsymbol{\tau}^{\top} & \ldots & \boldsymbol{\tau}^{\top}\end{array}\right]^{\top}$, of dimensions $\left(n_{\mathrm{tot}} \times 2\right)$, is the overall incidence matrix of the seismic ground motion; $\mathbf{U}(t)=$ $\left\{\left\{\mathbf{u}^{(0)}(t)\right\}^{\top}:\left\{\mathbf{u}^{(1)}(t)\right\}^{\top} \cdots \quad\left\{\mathbf{u}^{\left(n_{\mathrm{R}}\right)}(t)\right\}^{\top}\right\}^{\top}$ and $\mathbf{F}_{\mathrm{d}}(t)=\left\{\left\{\mathbf{f}_{\mathrm{d}}^{(1)}(t)\right\}^{\top} \cdots\left\{\mathbf{f}_{\mathrm{d}}^{\left(n_{\mathrm{R}}\right)}(t)\right\}^{\top}\right\}^{\top}$ are the arrays of the $n_{\text {tot }}$ DoFs of the coupled dynamic system and of the $n_{\text {Dtot }}$ reaction forces in the NLVDs, respectively. Combining Eqs. (10) and (11) for $j=1, \cdots, n_{\mathrm{R}}$, the latter can be expressed as:

$$
\mathbf{F}_{\mathrm{d}}(t)=\mathbf{C}_{\mathrm{d}} \cdot G_{\mathrm{d}}\left\{\boldsymbol{\Gamma}_{\mathrm{d}}^{\top} \cdot \dot{\mathbf{U}}_{\mathrm{d}}(t)\right\},
$$

in which $\mathbf{C}_{\mathrm{d}}$, of size $n_{\text {Dtot }}$, is the diagonal matrix of the viscous damper coefficients and $\boldsymbol{\Gamma}_{\mathrm{d}}$, of dimensions $\left(n_{\text {tot }} \times n_{\text {Dtot }}\right)$, is the matrix of the influence coefficients for all the NLVDs.

$$
\mathbf{C}_{\mathrm{d}}=\left[\begin{array}{ccc}
\mathbf{c}_{\mathrm{d}}^{(1)} & & \varnothing \\
& \ddots & \\
\varnothing & & \mathbf{c}_{\mathrm{d}}^{\left(n_{\mathrm{R}}\right)}
\end{array}\right] ; \quad \boldsymbol{\Gamma}_{\mathrm{d}}=\left[\begin{array}{ccc}
\gamma_{\mathrm{d}}^{(01)} & \cdots & \gamma_{\mathrm{d}}^{\left(0 n_{\mathrm{R}}\right)} \\
\hdashline \gamma_{\mathrm{d}}^{(1)} & & \varnothing \\
& \ddots & \\
\varnothing & & \gamma_{\mathrm{d}}^{\left(n_{\mathrm{R}}\right)}
\end{array}\right] .
$$

\section{CASE STUDY}

\subsection{Introduction}

The design concept and mathematical formulation illustrated in the previous sections have been applied to a real case study, namely to the seismic retrofitting of the so-called "Casa dello Studente", one of the largest student halls of accommodation owned by the University Messina, Italy, standing on a high-hazard earthquake zone (see Figure 2). The building has been subjected in recent years to many interventions to restore the architectural décor and to improve the services; unfortunately, as at today, all these interventions have been carried out in vain, as the building cannot be used until it meets the updated seismic safety criteria [1]. The proposed retrofitting approach is therefore aimed at increasing the level of seismic performance of the structure, without being overly invasive, so to preserve the previous non-structural interventions. The existing structure, with reinforced concrete (RC) frame and brick masonry infills, was built in the 1930s. The building has five stories $\left(n_{\mathrm{S}}=5\right)$, each one with an area of approximately $1,300 \mathrm{~m}^{2}$. The C-shaped plan of the building is symmetrical with respect to a NW-SE direction (assumed as $x$-direction in our computational model), and has a front side of $55 \mathrm{~m}$ and two wings of $37 \mathrm{~m}$. Three reaction towers $\left(n_{R}=3\right)$ are suggested for the seismic retrofitting of the building, namely a trapezoidal sub-frame (for architectural reasons) inside the courtyard, in the following referred to as the "third tower" $(j=3)$, and a rectangular sub-frame at the end of each wing, namely the "first tower" (on the NE side) and the "second tower" (on the SW side), as shown in Figure 3. The 


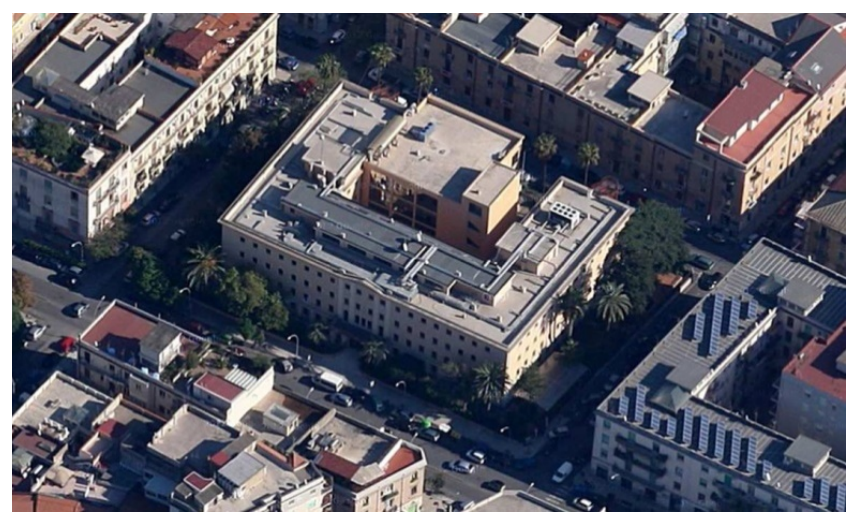

Figure 2. Aerial view of the case-study building

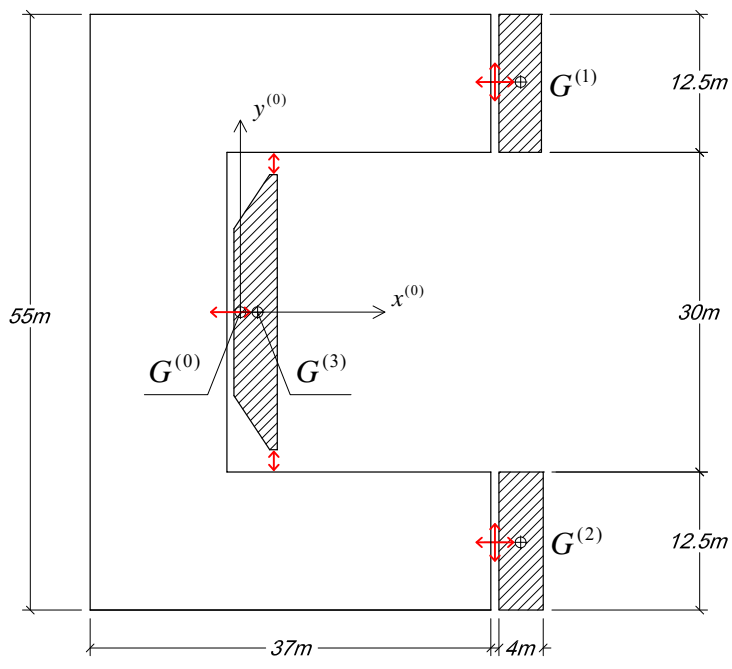

Figure 3. Plan of the building and reaction towers position; two-head arrows represent the lines of action of the NLVDs

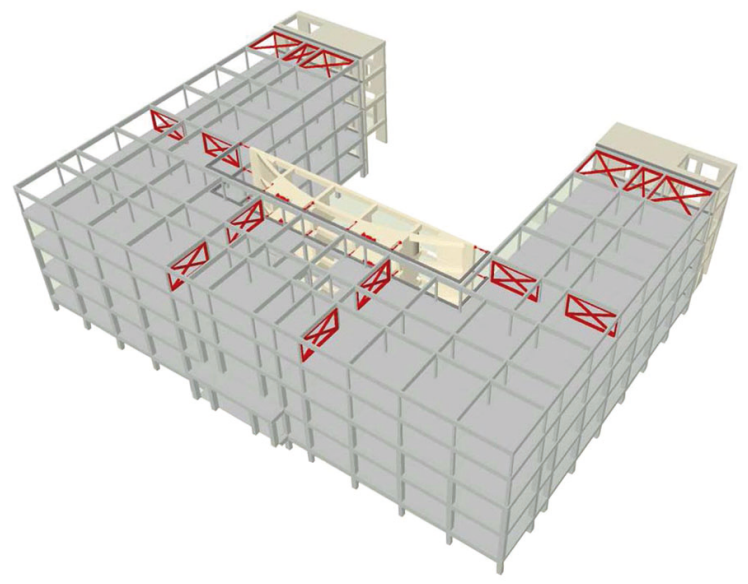

Figure 4.3D view of the FE model of building and towers; the bracing systems are needed to make the existing structure stronger and stiffer, so to resist the reaction forces of the NLVDs

three new towers are horizontally connected to the existing building through NLVDs in the $x$ and $y$ directions, whose resultant positions are identified by the two-head arrows in Figure 3. Steel spreaders will be required to avoid any localised damage at those positions.

\subsection{Structural parameters of the main building}

For the purposes of our numerical investigations, the existing building has been modelled with five rigid diaphragms in elevation, constant inter-story height $h=3.50 \mathrm{~m}$ and uniform distribution of mass and stiffness along the height (i.e. the existing building is regular in elevation). The lumped mass matrix has been defined assuming: translational mass $M^{(0)}=2.28 \times 10^{3} \mathrm{Mg}$; polar moment of inertia about the center of mass $J^{(0)}=1.04 \times 10^{6} \mathrm{Mg} \mathrm{m}^{2}$. The shear-type stiffness coefficients have been evaluated with the help of the commercial structural analysis program SAP2000 [33], including the bending flexibility of the beams and the additional stiffness provided by the masonry infills; a 3D view of the FE model is offered as Figure 4. The model in SAP2000 has also been used to validate the assumption of rigid diaphragm for the C-shaped floor of the existing building, as indeed the modal properties of the building showed only marginal changes when the rigid diaphragm was replaced with a slab. The values so obtained for the generic floor are: inter-story lateral stiffness in the $x$ direction $K_{x x}^{(0)}=9.375 \times 10^{6} \mathrm{kN} / \mathrm{m}$; in the $y$ direction $K_{y y}^{(0)}=8.625 \times 10^{6} \mathrm{kN} / \mathrm{m}$; 
Table I. Inclinations of the dampers [rad] and coordinates of their ends [m] with respect to the reference systems of existing building and reaction towers

\begin{tabular}{ccccccc}
$j$ & $\Theta_{\mathrm{d} 1}^{(j)}$ & $\Theta_{\mathrm{d} 2}^{(j)}$ & $X_{\mathrm{d} 1}^{(0 j)}=X_{\mathrm{d} 2}^{(0 j)}$ & $Y_{\mathrm{d} 1}^{(0 j)}=Y_{\mathrm{d} 2}^{(0 j)}$ & $X_{\mathrm{d} 1}^{(j)}=X_{\mathrm{d} 2}^{(j)}[\mathrm{m}]$ & $Y_{\mathrm{d} 1}^{(j)}=Y_{\mathrm{d} 2}^{(j)}$ \\
& {$[\mathrm{rad}]$} & {$[\mathrm{rad}]$} & {$[\mathrm{m}]$} & {$[\mathrm{m}]$} & {$[\mathrm{m}]$} & 0.00 \\
\hline 1 & 0 & $\pi / 2$ & 23.50 & 21.25 & -2.37 & 0.00 \\
2 & 0 & $\pi / 2$ & 23.50 & -21.25 & -2.37 & 0.00 \\
3 & 0 & $\pi / 2$ & 3.1 & 0.00 & 1.50 & 0.00 \\
\hline
\end{tabular}

torsional stiffness about the center of mass $K_{\varphi \varphi}^{(0)}=6.000 \times 10^{9} \mathrm{kN} \mathrm{m}$. Since the existing building is symmetric about the $x$ axis, the coupling stiffness coefficient $K_{x \varphi}^{(0)}$ is zero, while $K_{y \varphi}^{(0)}=-1.575 \times 10^{7} \mathrm{kN}$; furthermore, as all the lateral resisting members are either along $x$ or $y$, the coupling stiffness coefficient $K_{x y}^{(0)}$ is zero as well. With these values of mass and stiffness, the first three modal circular frequencies of the existing building disconnected from the towers are $\omega_{01}=17.39 \mathrm{rad} / \mathrm{s}, \omega_{02}=18.25 \mathrm{rad} / \mathrm{s}$ and $\omega_{03}=21.72 \mathrm{rad} / \mathrm{s}$. The damping matrix $\mathbf{c}^{(0)}$ has then been built so to have a constant damping ratio $\zeta^{(0)}=0.05$ for all the modes of vibration.

\subsection{Structural parameters of reaction towers}

Based on preliminary structural, architectural and economical considerations, the construction of three new towers, positioned as shown in Figures 3 and 4, has been deemed as a reasonable design solution for the proposed retrofitting strategy. The first two towers are identical, whereas the third one has a larger size. The story mass and polar moment of the towers are set as $M^{(j)}=M^{(0)} / 15$ and $J^{(j)}=J^{(0)} / 800$, respectively, with $j=1,2,3$. The lateral stiffness in both $x$ and $y$ directions for the first two towers are assumed to be proportional to the corresponding values in the existing building through the stiffness ratio $\lambda$, i.e. $K_{x x}^{(1)}=K_{x x}^{(2)}=\lambda K_{x x}^{(0)}$ and $K_{y y}^{(1)}=K_{y y}^{(2)}=\lambda K_{y y}^{(0)}$; for the third tower, which has a bigger size, the lateral stiffness is $50 \%$ higher, i.e. $K_{x x}^{(3)}=1.5 K_{x x}^{(1)}$ and $K_{y y}^{(3)}=1.5 K_{y y}^{(1)}$. The values of the torsional stiffenness are $K_{\varphi \varphi}^{(1)}=K_{\varphi \varphi}^{(2)}=(\lambda / 16) K_{\varphi \varphi}^{(0)}$ and $K_{\varphi \varphi}^{(3)}=1.5 K_{\varphi \varphi}^{(1)}$, while all the lateral-torsional coupling coefficients are zero, i.e. $K_{x \varphi}^{(j)}=K_{y \varphi}^{(j)}=0$ for $j=1,2,3$. The first three modal circular frequencies of the towers are: $\omega_{11}=\omega_{21}=\omega_{31} / \sqrt{1.5}=(67.80 \sqrt{\lambda}) \mathrm{rad} / \mathrm{s} ; \omega_{12}=\omega_{22}=\omega_{32} / \sqrt{1.5}=(70.69 \sqrt{\lambda}) \mathrm{rad} / \mathrm{s} ; \omega_{13}=\omega_{23}=\omega_{33} / \sqrt{1.5}=$ $(152.87 \sqrt{\lambda}) \mathrm{rad} / \mathrm{s}$. As in the case of the existing building, all the modes of vibration have been used $\left(m_{j}=3 n_{\mathrm{S}}\right)$ to build the matrices of inherent damping $\mathbf{c}^{(j)}$ (with $j=1,2,3$ ), assuming the same modal viscous damping ratio $\zeta^{(j)}=0.05$. It is worth noting here that the coefficients $M^{(0)} / M^{(j)=15}, J^{(0)} / J^{(j)=800}$ and $K^{(3)} / K^{(1)=1.5}$ have been assumed based on preliminary design considerations on the individual reaction towers and the feasibility of the intervention; these values could be potentially be further optimised through appropriate heuristic search methods (e.g. genetic algorithms and particle swarm optimisation, as in Refs. [34, 35]), but this beyond the scope of the present study.

\subsection{Layout of the NLVDs}

For the generic level, two NLVDs connect in the $x$ and $y$ directions the main building to each tower. The orientation and location of the devices is fully defined in Table I. It is worth noting here that:

- the two devices connecting main building and third tower in the $y$ direction share the same line of action (see Figure 3) and therefore a single NLVD with twice the viscous damper coefficient can be used in the mathematical model;

- the position of the devices has not been optimized, but chosen considering the architectural and service constraints;

- if $n_{\mathrm{S}}^{*}$ is the number of stories in which the main building is connected to the reaction towers (with $1 \leq n_{\mathrm{S}}^{*} \leq n_{\mathrm{S}}$ ), then the number of seismic devices for each tower is $n_{\mathrm{D} j}=2 n_{\mathrm{S}}^{*}($ with $j=1,2,3)$, and in total $n_{\text {Dtot }}=6 n_{\mathrm{S}}^{*}$. 


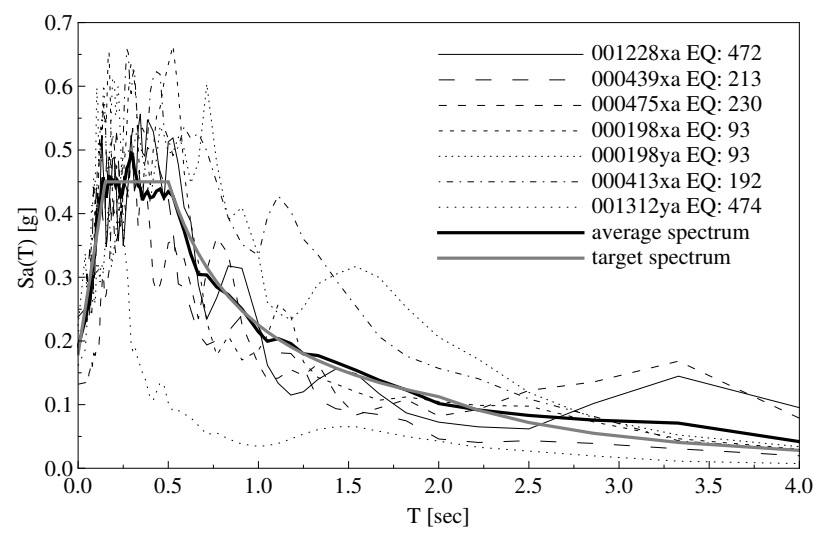

(a)

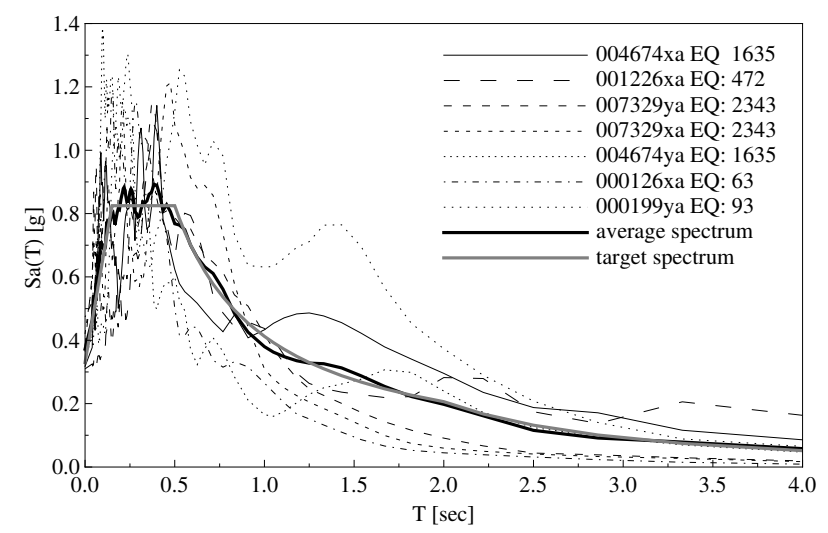

(b)

Figure 5. Response spectra of the recorded accelerograms used for DLR (a) and NCR (b) scenarios

\subsection{Numerical analyses}

The numerical results reported in the following are consistent with the methods of nonlinear dynamic analysis suggested by Eurocode 8 [36], and as such they have been obtained with time-history simulations carried out with two sets of seven recorded accelerograms each, named "set 1" and "set 2", representing two different levels of seismic hazard. The 4th-order explicit Runge-Kutta method has been adopted to integrate numerically the equations of motion [37]. The freeware program REXEL [38] has been used to select the records from the European Strong Motion Data [39] and match (with $\pm 10 \%$ of tolerance) the pertinent Eurocode 8 target spectra [36] without any scaling. Considering the geographic location and ground conditions of the site: $i$ ) soil class "B" was chosen for the definition of the seismic input (corresponding to "deposit of very dense sand" and soil amplification factor $S=1.2$ ); $i i$ ) the design values of the peak ground acceleration (PGA) at bedrock (reference soil class "A") were taken as $a_{\mathrm{g}}=0.150 \mathrm{~g}$ for set 1 ( $g=9.81 \mathrm{~m} / \mathrm{s}^{2}$ being the acceleration of gravity) and $a_{\mathrm{g}}=0.275 \mathrm{~g}$ for set $2(+83.3 \%)$, which correspond to the "Damage Limitation Requirement" (DLR) and "No-Collapse Requirement" (NCR), respectively. The 5\%-damping elastic response spectra for the accelerograms of sets 1 and 2 are shown in Figures 5a and 5b, respectively, along with their "waveform" and "earthquake" id codes; target spectra and average spectra are also plotted. In order to compare the results of the "retrofitted" configuration (with NLVDs connecting existing building and reaction towers) with those of the "unconnected" configuration (without dampers), two performance indexes have been defined:

- Shear index $S I^{(j)} \quad[\%]$, as the ratio between the mean value of the maximum base shear $\bar{V}_{\max }^{(j)}=$ $\overline{\max \left\{\left|V^{(j)}(t)\right|\right\}}$ experienced by the $j$ th sub-frame in the retrofitted and unconnected configurations, where the overline means the average over the (seven) earthquake records used for a given seismic analysis; furthermore, the symbols $S I^{(\mathrm{T})}$ and $\bar{V}_{\max }^{(\mathrm{T})}$ refer to the resultant of the shear forces at the base of all the reaction towers, i.e. $V^{(\mathrm{T})}(t)=\sum_{j=1}^{n_{\mathrm{R}}} V^{(j)}(t)$, while $S I^{(\mathrm{T})}$ is calculated with respect to the case where the reaction towers are disconnected, i.e. when all damper coefficients $c_{\mathrm{d} k}^{(j)} \rightarrow 0$;

- Displacement index $D I^{(j)}[\%]$, as the retrofitted-to-unconnected ratio between the mean values of the maximum displacements at any one corner node of the top story of the $j$ th sub-frame, $\bar{u}_{\max }^{(j)}$.

Importantly: $i$ ) the above performance indexes depend on the particular direction along which base shear $V$ and displacement $u$ are calculated (this is particularly important if torsional effects are induced by the seismic input); ii) the demand parameters $\bar{V}_{\max }^{(j)}, \bar{V}_{\max }^{(\mathrm{T})}$ and $\bar{u}_{\max }^{(j)}$ represent the average of the seven maxima calculated for the seven records of each set. For a better ease of comparison, Table II reports the performance indexes $S I^{(0)}$ and $D I^{(0)}$ computed in all the 24 scenarios better described in the following subsections, along with the corresponding values of $\bar{V}_{\max }^{(j)}, \bar{V}_{\max }^{(\mathrm{T})}$ and the optimal viscous damper coefficient $c_{\mathrm{d} \text {,opt }}$ (which minimizes $\bar{V}_{\max }^{(0)}$ ). 
Table II. Performance indicators for the various numerical simulations

\begin{tabular}{|c|c|c|c|c|c|c|c|c|c|}
\hline$\alpha_{\mathrm{d}}$ & $\lambda$ & Connections & Set & $\begin{array}{c}\Theta_{\mathrm{g}} \\
{[\mathrm{rad}]}\end{array}$ & $\begin{array}{l}c_{\mathrm{d}, \mathrm{opt}} \\
{[\mathrm{kN}]}\end{array}$ & $S I_{\min }^{(0)}$ & $D I_{\min }^{(0)}$ & $\begin{array}{c}V_{\max }^{(0)} \\
{[\mathrm{MN}]}\end{array}$ & $\begin{array}{l}V_{\max }^{(\mathrm{T})} \\
{[\mathrm{MN}}\end{array}$ \\
\hline 0.15 & $1 / 6$ & All stories & 1 & 0 & $10^{2.8}$ & 0.73 & 0.73 & 31.8 & 13.9 \\
\hline 0.30 & $"$ & $"$ & $"$ & $"$ & $10^{2.9}$ & 0.72 & 0.72 & 31.5 & 13.7 \\
\hline 0.60 & $"$ & $"$ & $"$ & $"$ & $10^{3.3}$ & 0.71 & 0.71 & 31.3 & 13.9 \\
\hline 1.00 & $"$ & $"$ & $"$ & $"$ & $10^{3.7}$ & 0.71 & 0.70 & 31.2 & 13.0 \\
\hline 0.15 & $"$ & $"$ & 2 & $"$ & $10^{3.1}$ & 0.70 & 0.70 & 56.5 & 29.8 \\
\hline 0.30 & $"$ & $"$ & $"$ & $"$ & $10^{3.3}$ & 0.70 & 0.70 & 56.4 & 29.8 \\
\hline 0.60 & $"$ & $"$ & $"$ & $"$ & $10^{3.6}$ & 0.70 & 0.70 & 56.6 & 28.8 \\
\hline 1.00 & $"$ & $"$ & $"$ & $"$ & $10^{4.1}$ & 0.70 & 0.69 & 57.0 & 29.0 \\
\hline 0.15 & $"$ & $"$ & $2 \mathrm{D}$ & $"$ & $10^{2.9}$ & 0.70 & 0.70 & 30.8 & 16.4 \\
\hline$"$ & $"$ & $"$ & $1 \mathrm{U}$ & $"$ & $10^{3.0}$ & 0.73 & 0.74 & 58.4 & 27.3 \\
\hline 1.00 & $"$ & $"$ & $2 \mathrm{D}$ & $"$ & $10^{4.1}$ & 0.71 & 0.68 & 31.1 & 15.8 \\
\hline$"$ & $"$ & $"$ & $1 \mathrm{U}$ & $"$ & $10^{3.7}$ & 0.71 & 0.70 & 57.2 & 23.8 \\
\hline 0.15 & $1 / 4$ & $"$ & 1 & $"$ & $10^{3.0}$ & 0.60 & 0.60 & 26.1 & 19.0 \\
\hline$"$ & $1 / 8$ & $"$ & $"$ & $"$ & $10^{2.5}$ & 0.81 & 0.82 & 35.4 & 11.8 \\
\hline$"$ & $1 / 4$ & $"$ & 2 & $"$ & $10^{3.3}$ & 0.60 & 0.59 & 48.0 & 37.4 \\
\hline$"$ & $1 / 8$ & $"$ & $"$ & $"$ & $10^{2.9}$ & 0.81 & 0.80 & 65.1 & 25.4 \\
\hline$"$ & $1 / 6$ & 1st, 5th & $"$ & $"$ & $10^{3.5}$ & 0.71 & 0.71 & 57.6 & 29.4 \\
\hline$"$ & $"$ & $3 \mathrm{rd}, 5$ th & $"$ & $"$ & $10^{3.3}$ & 0.74 & 0.70 & 59.4 & 26.2 \\
\hline$"$ & $"$ & 4 th, 5 th & $"$ & $"$ & $10^{3.2}$ & 0.75 & 0.72 & 60.4 & 24.4 \\
\hline$"$ & $"$ & 1st, 2nd, 3rd & $"$ & $"$ & $10^{3.5}$ & 0.70 & 0.76 & 56.7 & 31.4 \\
\hline$"$ & $"$ & $3 \mathrm{rd}, 4$ th, 5 th & $"$ & $"$ & $10^{3.1}$ & 0.74 & 0.70 & 59.4 & 25.9 \\
\hline 0.15 & $1 / 4$ & All stories & $"$ & $\pi / 2$ & $10^{3.3}$ & 0.59 & 0.66 & 49.3 & 33.4 \\
\hline$"$ & $1 / 6$ & $"$ & $"$ & $"$ & $10^{3.0}$ & 0.72 & 0.76 & 59.5 & 26.9 \\
\hline$"$ & $1 / 8$ & $"$ & $"$ & $"$ & $10^{2.9}$ & 0.80 & 0.81 & 66.2 & 24.6 \\
\hline
\end{tabular}

\subsection{Influence of the velocity exponent $\alpha_{d}$}

The effects of the degree of nonlinearity of the viscous dampers on the structural behavior was first investigated by considering four values of the velocity exponent, namely: $\alpha_{\mathrm{d}}=0.15,0.30,0.60$ and 1.00 (the latter case corresponding to linear viscous dampers). The analysis also assumed:

- ground shaking along the $x$ axis only $\left(\Theta_{\mathrm{g}}=0\right)$, meaning that, due to the symmetry of the problem, all structures experience seismic movements in the $x$ direction only (and therefore only the NLVDs in the $x$ direction are significant);

- stiffness ratio $\lambda=1 / 6$;

- $n_{\mathrm{S}}^{*}=n_{\mathrm{S}}$, i.e. existing building and reaction towers are connected at all floors.

The key outcomes of the time-history analyses are summarized by Figures 6a and 6b, which show $\bar{V}_{\max }^{(0)}$ and $\bar{V}_{\max }^{(\mathrm{T})}$ for sets 1 and 2, respectively, versus the viscous damper coefficient $c_{\mathrm{d}}$ and Figures $7 \mathrm{a}$ and $7 \mathrm{~b}$, which plot the mean value of the maximum force $\bar{f}_{\mathrm{d}, \max }$ exerted by the damper connecting the top story of the existing building to 3rd tower, again for sets 1 and 2. In all these log-linear graphs: $i$ ) the horizontal axis spans the values of $c_{\mathrm{d}}$ between 4 and $30,000 \mathrm{kN} ; i i$ ) the thick solid lines connect the data points obtained for the optimal value of the viscous damper 


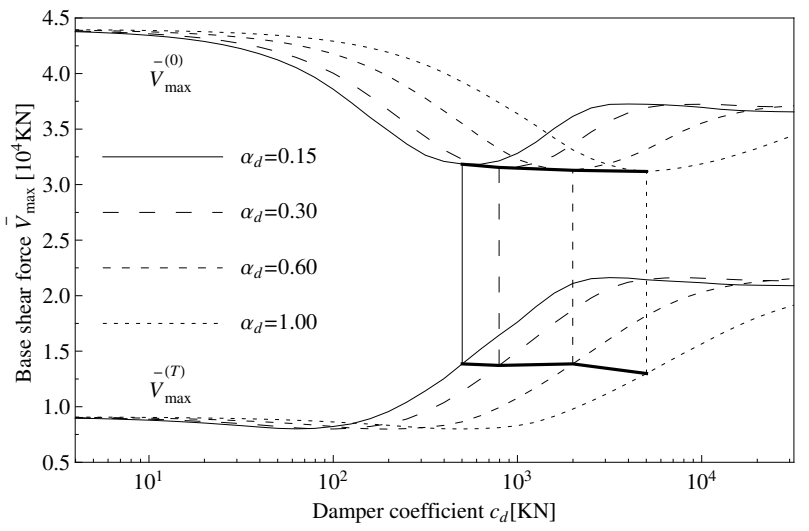

(a)

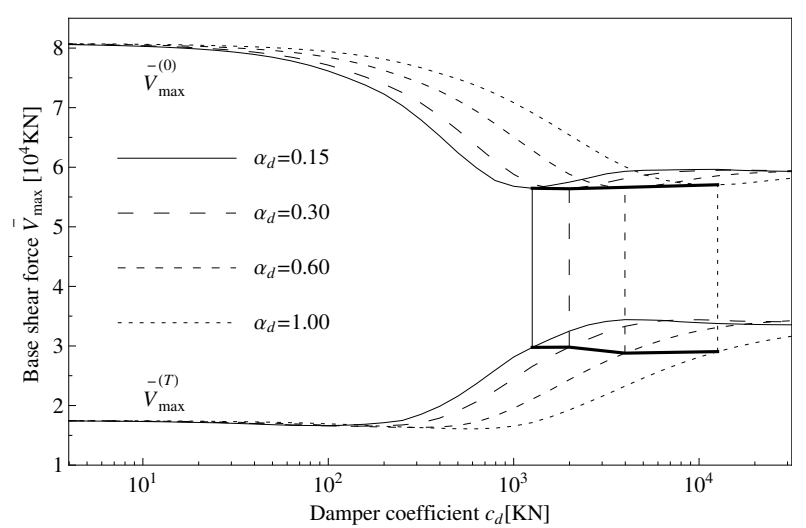

(b)

Figure 6. Maximum base shear vs. viscous damper coefficient for the stiffness ratio $\lambda=1 / 6$, different velocity exponents $0.15 \leq \alpha_{\mathrm{d}} \leq 1.00$, seismic input in the $x$ direction and both DLR (a) and NCR (b) scenarios; the thick lines show the maximum base shear on the existing building and the three towers for the optimal viscous damper coefficients

coefficient, $c_{\mathrm{d}, \mathrm{opt}}$, for which the maximum reduction in the base shear force of the existing building has been achieved. Interestingly:

- for $c_{\mathrm{d}}=0$, the main building is disconnected from the reaction towers, and this corresponds to the existing un-retrofitted conditions $\left(S I^{(0)}=D I^{(0)}=1\right)$;

- for $c_{\mathrm{d}} \rightarrow+\infty$, small relative velocities between the main building and the reaction towers result into large forces in the seismic devices, meaning the four sub-frames behave as if they were rigidly connected;

- for a given value of $\alpha_{\mathrm{d}}$, the optimal viscous damper coefficient $c_{\mathrm{d} \text {,opt }}$ exploits the coupled dynamics of the sub-frames in order to reduce the seismic effects on the main building.

For both excitation levels, increasing the velocity exponent $\alpha_{\mathrm{d}}$ does not affect significantly the capability of the damper to reduce the base shear force in the main building (see the top, almost flat thick lines in Figures 6a and $6 \mathrm{~b}$ ) and the amount of shear force transferred to the reaction towers (see the bottom thick lines in the same plots); however, a remarkable increment is observed in the optimal viscous damper coefficients $c_{\mathrm{d} \text {,opt }}$ (almost an order of magnitude higher in the logarithmic scale of the horizontal axes in all the four plots) and in the maximum forces in the dampers (see the variation in the ordinates of the thick lines in Figures $7 \mathrm{a}$ and $7 \mathrm{~b}$ ). As an example, for $\alpha_{\mathrm{d}}=0.15$, which is the smallest velocity exponent that can be found in practice, the minimum shear indexes and the optimal coefficients (for which the minimum is achieved) are: $S I_{\min }^{(0)}=0.73$ and $c_{\mathrm{d}, \mathrm{opt}}=10^{2.8} \mathrm{kN}$ for set $1 ; S I_{\min }^{(0)}=0.70$ and $c_{\mathrm{d}, \mathrm{opt}}=10^{3.1} \mathrm{kN}$ for set 2 ; while the corresponding increments in the total shear forces experienced by all the reaction towers are $S I^{(\mathrm{T})}=1.55$ for set 1 and $S I^{(T)}=1.71$ for set 2 . For $\alpha_{\mathrm{d}}=1$ (linear viscous dampers), the same parameters become: $S I_{\min }^{(0)}=0.71, S I^{(\mathrm{T})}=1.43\left(8 \%\right.$ reduction) and $c_{\mathrm{d}, \mathrm{opt}}=10^{3.7} \mathrm{kN}$ (about $700 \%$ increase) for set 1 ; $S I_{\text {min }}^{(0)}=0.70, S I^{(\mathrm{T})}=1.66\left(3 \%\right.$ reduction) and $c_{\mathrm{d}, \mathrm{opt}}=10^{4.1} \mathrm{kN}$ (massive $900 \%$ increase) for set 2 . As a consequence, also the maximum force $\bar{f}_{\mathrm{d} \text {,max }}$ for the optimal viscous damper coefficient $c_{\mathrm{d} \text {,opt }}$ increases in the monitored damper when the velocity exponent $\alpha_{\mathrm{d}}$ increases: specifically, from 399 to $869 \mathrm{kN}$ for set $1(+73 \%)$; from 1, 084 to $2,470 \mathrm{kN}$ for set $2(+128 \%)$.

It is interesting to observe that, independently of the level of the seismic input and the set of accelerograms used, the same trend of results has been found. In particular, the lowest value of velocity exponent $\alpha_{\mathrm{d}}$ always provides the better performance, meaning that the nonlinearity of the viscous dampers increases their efficiency. Furthermore, as expected, the stronger the earthquake, the larger the optimal viscous damper coefficients $c_{\mathrm{d} \text {,opt }}$, the larger and more expensive the devices. In the design stage, therefore, preference should be given to highly nonlinear devices (small value of $\alpha_{\mathrm{d}}$ ) with the viscous damper coefficient taken as $c_{\mathrm{d} \text {,opt }}$ for the NCR analyses, checking afterwards that the performance of the retrofitted building is also acceptable for the DLR. Incidentally, one can also note that buildingtower rigid connections $\left(c_{\mathrm{d}} \rightarrow+\infty\right)$ always deliver larger values of both $S I^{(0)}$ and $S I^{(\mathrm{T})}$ with respect to $c_{\mathrm{d}, \text { opt }}$. For instance, assuming $c_{\mathrm{d}} \rightarrow+\infty: S I^{(0)}=0.84$ and $S I^{(\mathrm{T})}=2.33$ for set $1 ; S I^{(0)}=0.75$ and $S I^{(\mathrm{T})}=1.93$ for set 2 . This corresponds, on average, to a performance loss of $13 \%$ for the main building and $32 \%$ for the reaction towers, which 


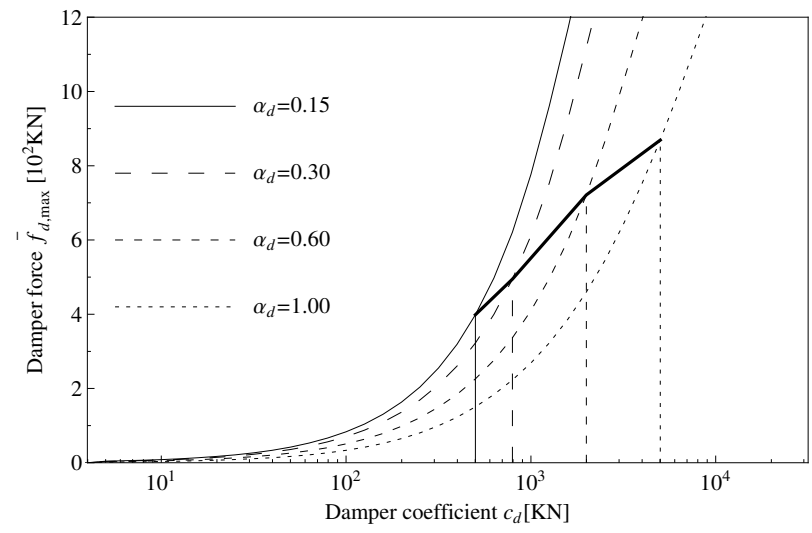

(a)

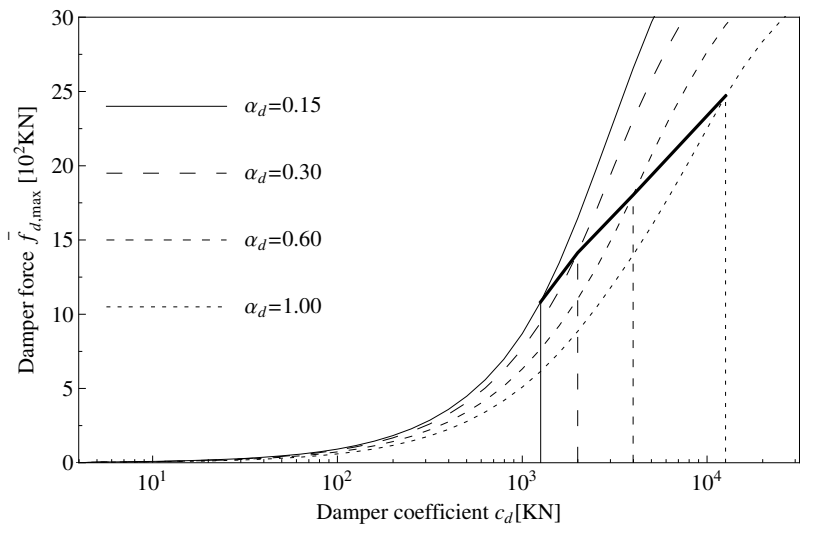

(b)

Figure 7. Variation with $c_{\mathrm{d}}$ of the maximum damper force between existing building and third reaction tower at the top floor for the stiffness ratio $\lambda=1 / 6$, different velocity exponents $0.15 \leq \alpha_{\mathrm{d}} \leq 1.00$, seismic input in the $x$ direction and both DLR (a) and NCR (b) scenarios; the thick lines show the maximum damper force for optimal viscous damper coefficients

demonstrates the importance of selecting the optimum value for the viscous damper coefficient $c_{\mathrm{d}}$, and the significant loss of efficiency that can happen when the devices are oversized.

The results presented above also allow identifying similarities and differences between the proposed retrofitting strategy and a more conventional approach in which the NLVDs are mounted into an existing structure with steel chevron braces (e.g. Ref. [22]). In both cases, the lower the velocity exponent, the lower the maximum forces in the devices. However, while for devices mounted internally the structural performance tends to deteriorate when the degree of nonlinearity increases, this does not happen in the proposed approach, in which the velocity exponent does not affect significantly $\bar{V}_{\max }^{(0)}$ for $c_{\mathrm{d}, \mathrm{opt}}$.

\subsection{Modal properties for the case of linear viscous dampers}

To complement the results of the nonlinear time-history analyses presented in the previous subsection, the effects of the tower-damper retrofitting intervention are analysed in this subsection with the modal analysis for the linear case, i.e. $\alpha_{\mathrm{d}}=1$, and again considering $\lambda=1 / 6$ and the seismic input in the $x$ direction. Specifically, the variation of modal frequencies and modal damping ratios has been tracked for increasing values of $c_{\mathrm{d}}$. The results of this analysis are depicted in Figures $8 \mathrm{a}$ and $8 \mathrm{~b}$, respectively, for the five modes of vibration which have the larger values of modal participation mass coming from the existing building, and the trends are aligned with those highlighted by Zhang and $\mathrm{Xu}[12]$.

For very low values of the damper coefficient, say $c_{\mathrm{d}}<10^{3} \mathrm{kN}$ for the case-study building, it is observed that the modes of vibration are those of the unconnected structures, and thus the coupled building-damper-tower system can be considered as classically damped, as existing building and reaction towers vibrate independently of each other. In the opposite limiting case, when the damper constant takes very large values, say $c_{\mathrm{d}}>5 \times 10^{5} \mathrm{kN}$, the structures behave as if they were rigidly connected; accordingly, the total number of modes of vibration tends to reduce to five (i.e. one for each floor for $c_{\mathrm{d}} \rightarrow+\infty$ ) and, due to uniform distribution of masses, lateral-resisting elements and dampers along the height, the non-classical part of the viscous damping matrix is negligible. In the intermediate range of values for $c_{\mathrm{d}}$, however, the coupled dynamic system becomes non-classically damped.

If the first mode of vibration is considered (solid lines), a maximum damping ratio $\zeta_{1, \text { new }}=0.127$ is achieved for $c_{\mathrm{d}}=10^{3.5} \mathrm{kN}$, a value lower than the optimal value $c_{\mathrm{d} \text {,opt }}$ found through the nonlinear time-history analyses. Specifically (see Table II), considering $\lambda=1 / 6$ and $\alpha_{\mathrm{d}}=1$, set 1 of time-history seismic analyses delivers an optimal value of $c_{\mathrm{d}}$ which is $10^{(3.7-3.5)} \approx 1.6$ times larger than the value obtained through a complex-valued modal analysis; for set 2 the discrepancy is even higher, as the optimal value of $c_{\mathrm{d}}$ is $10^{(4.1-3.5)} \approx 4.0$ times larger. Interestingly, owing to the linearity of the combined structural system in these analyses, this difference is only due to the frequency content and the non-stationarity of the recorded accelerograms selected for the two earthquake sets.

Figure 9 shows the real and imaginary parts of the first modal shape for all the individual structures. The nonclassical nature of the modal damping is confirmed in this case by the relatively large magnitude of the imaginary part 


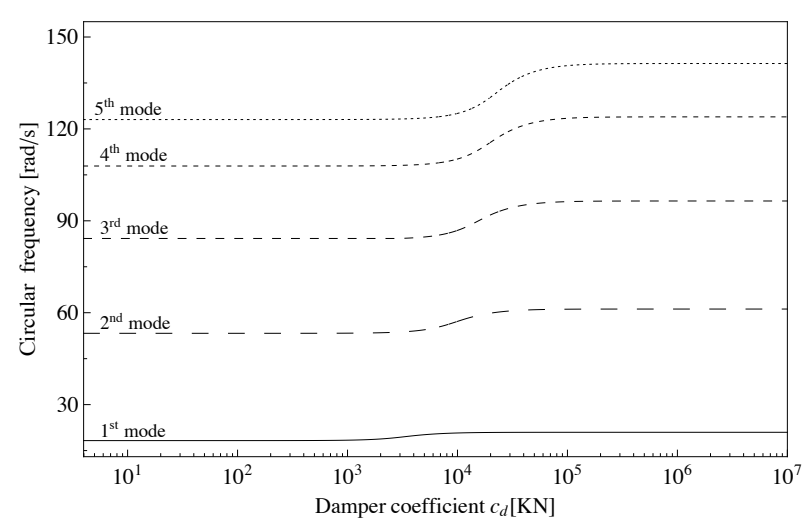

(a)

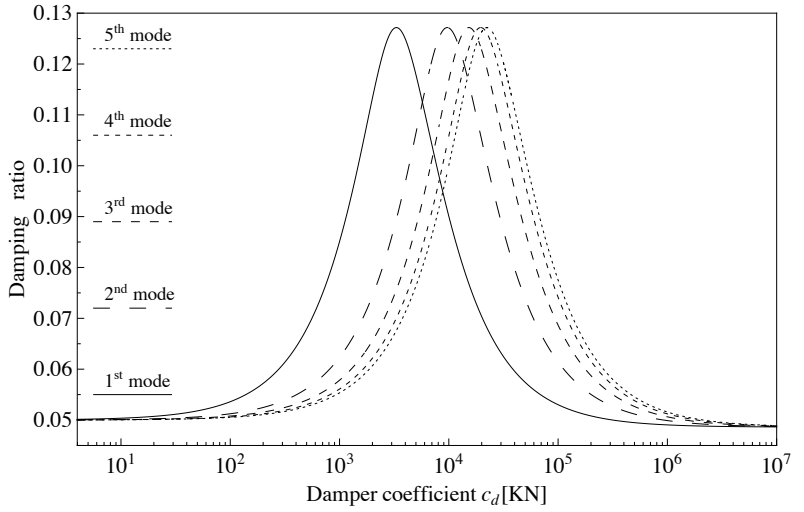

(b)

Figure 8. Tracking the variation with $c_{\mathrm{d}}$ of the modal frequencies (a) and modal viscous damping ratios (b) of the modes of vibration with more modal mass coming from the main structure for the case of linear viscous dampers $\left(\alpha_{\mathrm{d}}=1\right)$, with stiffness ratio $\lambda=1 / 6$ and seismic input in the $x$ direction.

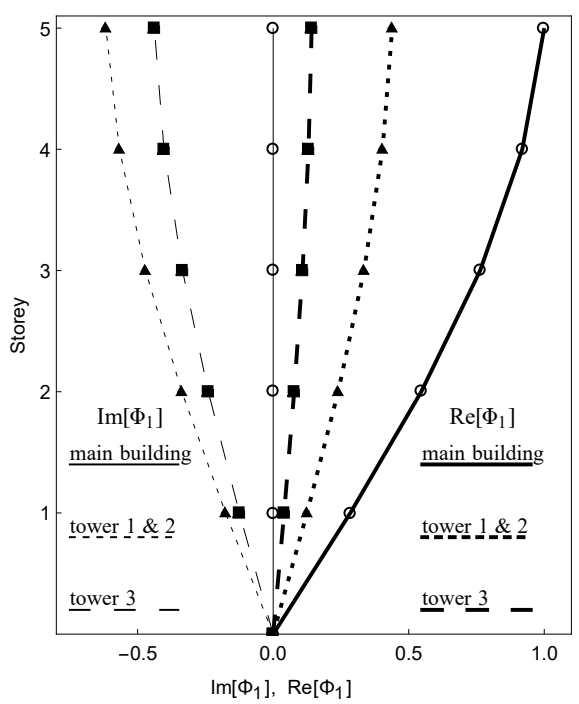

Figure 9. Real and imaginary part of the first complex-valued mode of vibration along the $x$ direction for $\lambda=1 / 6, \alpha_{\mathrm{d}}=1$ and

$$
c_{\mathrm{d}}=10^{3.5}
$$

(thin lines) for the towers 1, 2 and 3 when compared to their real part (thick lines). The corresponding modal circular frequency is $\omega_{1, \text { new }}=18.5 \mathrm{rad} / \mathrm{s}$, about $7 \%$ higher than the fundamental frequency of the existing building along the $x$ direction when disconnected from the auxiliary reaction towers.

\subsection{Influence of seismic input}

The results presented in the previous subsection clearly show that the optimal viscous damper coefficient $c_{\mathrm{d}, \mathrm{opt}}$ increases with the level of the seismic input. Since the accelerograms of sets 1 and 2 have not been scaled, i.e. different records have been used for the two design scenarios of DLR and NCR, this effect could be the consequence of: $i$ ) the different time-frequency characteristics of the accelerograms selected for the two sets; $i i$ ) the nonlinearity associated with the viscous dampers. In order to quantify the direct effects of the nonlinearity, which is of more general relevance in the present study, two further sets of seismic analyses were carried out with accelerograms derived by upscaling those of set 1 by a factor of $1.833=0.275 / 0.150$ and downscaling those of set 2 by the same factor (which is the ratio 
of $a_{\mathrm{g}}$ between the corresponding elastic design spectra). In this way the new "set 1U" (upscaled set 1) and "set 2D" (downscaled set 2) match the NCR and DLR scenarios, respectively, so that:

- any difference in the values of $S I_{\min }^{(0)}$ and $c_{\mathrm{d} \text {,opt }}$ between sets 1 and $1 \mathrm{U}$, or between sets 2 and $2 \mathrm{D}$, only depends on the nonlinearity of the viscous dampers;

- the sensitivity of the retrofitting strategy to the selection of the accelerograms would appear by comparing the numerical results for sets 1 and 2D (for the DLR) and sets 2 and $1 \mathrm{U}$ (for the NCR).

Only the extreme values of the velocity exponent were considered for this analysis, namely $\alpha_{\mathrm{d}}=0.15$ and $\alpha_{\mathrm{d}}=1.00$, corresponding to highly-nonlinear and linear viscous dampers, while $\lambda=1 / 6$ was kept constant. As expected, the results for the linear cases are marginally affected by the choice of the seven accelerograms matching the same elastic design spectrum, as evident by comparing the relevant curves in Figures 10a and 10b. Conversely, more pronounced differences appear for the nonlinear cases, particularly for higher values of the viscous damping constant.

Interestingly, while the variation of $c_{\mathrm{d}, \text { opt }}$ does not involve a significant change in the reduced seismic forces on the main building, i.e. similar values of $S I_{\min }^{(0)}$ are obtained, its effects on the force exerted to the reaction towers can be much larger. For this reason, considering the random nature of the seismic events, it could be advisable at the design stage to adopt a value of $c_{\mathrm{d}}$ slightly less than $c_{\mathrm{d} \text {,opt }}$, as the loss of effectiveness in the earthquake protection of the main building might be relatively small (i.e. the function $\bar{V}^{(0)}\left(c_{\mathrm{d}}\right)$ tends to be quite flat about its minimum), but a considerable reduction in the seismic actions on the reaction tower could be obtained (as conversely the function $\bar{V}^{(\mathrm{T})}\left(c_{\mathrm{d}}\right)$ is always quite steep for $\left.c_{\mathrm{d}}=c_{\mathrm{d}, \mathrm{opt}}\right)$.

Figure 11 highlights the effects of the dampers' nonlinearity on the seismic response of both the main building and the reaction towers through the ratios of $\bar{V}_{\max }^{(0)}$ and $\bar{V}_{\max }^{(\mathrm{T})}$ when the amplitude of the accelerograms is increased by the NCR/DLR ratio of 1.833 , while keeping constant $\alpha_{\mathrm{d}}=0.15$ and $\lambda=1 / 6$. As expected, in the case of a linear behavior, i.e for $c_{\mathrm{d}}=0$ (when the sub-frames are disconnected), $c_{\mathrm{d}} \rightarrow+\infty$ (when the sub-frames become rigidly connected), or $\alpha_{\mathrm{d}}=1$ (i.e. linear viscous dampers are installed), the ratios of $\bar{V}_{\max }^{(0)}$ and $\bar{V}_{\max }^{(\mathrm{T})}$ for both sets 1 and $1 \mathrm{U}$ and sets 2D and 2 coincide with the ratio of the seismic input (represented by the reference horizontal line of ordinate 1.833). Conversely, in the range of practical interest for the viscous damper coefficients, the nonlinearity can affect significantly the structural response. The reaction towers are more sensitive to the nonlinear behavior of the viscous dampers, showing a reduction with respect to the linear case that can be as large as $20 \%$. The effects on the main building are less pronounced, with variations of about $\pm 10 \%$ in comparison to the linear case and a reduction of $\bar{V}_{\max }^{(0)}$ only seen for relatively large values of $c_{\mathrm{d}}$. Furthermore, if the dampers are designed with the value of $c_{\mathrm{d}}$ optimized for the NCR, the seismic input for the DLR scenario will result in an increase in the maximum damper force with respect to the design value directly optimized for the DLR, namely from $399 \mathrm{kN}$ to $961 \mathrm{kN}$ for $\alpha_{\mathrm{d}}=0.15$ and from $869 \mathrm{kN}$ to $1,407 \mathrm{kN}$ for $\alpha_{\mathrm{d}}=1$. Unsurprisingly, due to the constitutive law of the damper, the percentage increase in the damper force is higher for the nonlinear case $(+141 \%)$ than for the linear one $(+62 \%)$. For both cases anyway the maximum damper force is lower than that evaluated for the NCR scenario (respectively equal to $1,084 \mathrm{kN}$ and $2,470 \mathrm{kN}$ ).

\subsection{Influence of the stiffness ratio $\lambda$}

The elastic stiffness of the reaction towers is one of the most crucial design parameter, as it directly affects the performance of the proposed retrofitting strategy. Clearly, a combination of very stiff tower $(\lambda \gg 1)$ and rigid connections $\left(c_{\mathrm{d}} \rightarrow+\infty\right)$ is theoretically capable of reducing the seismic-induced displacements in the existing building to any desired value. In practical design situations, however, the dimensions of the reaction towers should be compatible with those of the existing building, so that the stiffness of the new sub-frames is limited, and it can be realistically assumed $\lambda<1 / 2$, if not less. Furthermore, the construction of the reaction towers, including their foundations, represents the major cost in the proposed retrofitting intervention, and this cost obviously increases with their stiffness.

To quantify the effects that different values of the stiffness of the reaction towers can have on the case study under investigation, three design scenarios have been analyzed, corresponding to the stiffness ratios $\lambda=1 / 4,1 / 6$ and $1 / 8$, which in the following are ordinately referred to as "stiff", "medium" and "flexible" towers. As in the previous subsection, the $x$ axis is the direction of attack of the ground shaking $\left(\Theta_{\mathrm{g}}=0\right)$ and $n_{\mathrm{S}}^{*}=n_{\mathrm{S}}$, while only the low velocity exponent $\alpha_{\mathrm{d}}=0.15$ has been used for this set of numerical simulations, as this value has consistently shown the best performance in all previous analyses. 


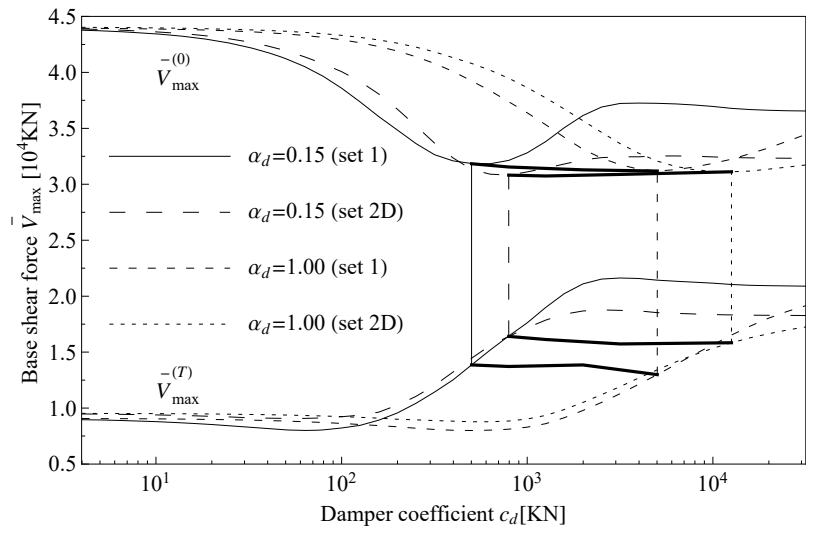

(a)

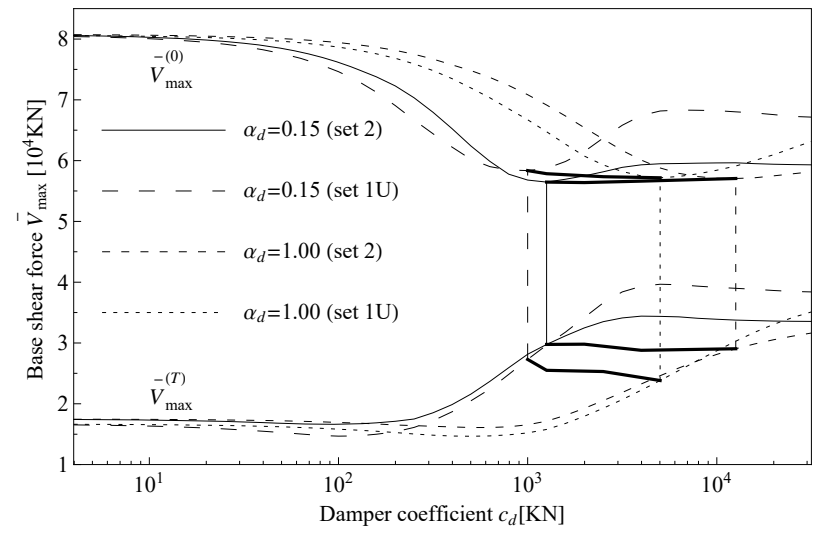

(b)

Figure 10. Maximum base shear vs. viscous damper coefficient for the linear $\left(\alpha_{\mathrm{d}}=1\right)$ and nonlinear $\left(\alpha_{\mathrm{d}}=0.15\right)$ behavior, stiffness ratio $\lambda=1 / 6$, seismic input in $x$ direction and two sets of accelerograms matching the DLR (a) and NCR (b) scenarios; the thick lines show the maximum base shear on the existing building and the three towers for the optimal viscous damper coefficients

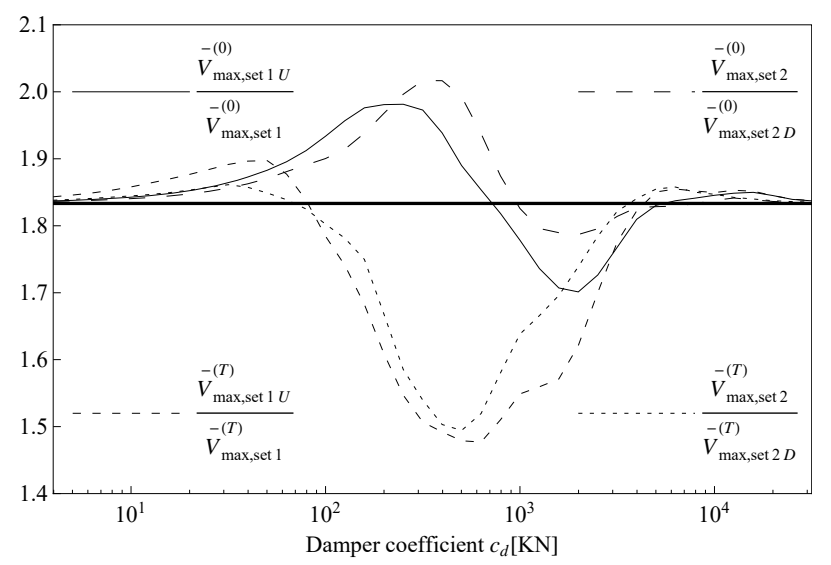

Figure 11. Ratio of the maximum base shear forces computed for the same set of $x$-direction accelerograms, stiffness ratio $\lambda=1 / 6$ and nonlinear damping exponent $\alpha_{\mathrm{d}}=0.15$, but different intensities of the seismic input; the straight thick line is for the intensity ratio $\mathrm{NCR} / \mathrm{DLR}=1.833$

For set 1 (see Figure 12a), the minimum shear index and the optimal damper constant are: $S I_{\min }^{(0)}=0.60$ and $c_{\mathrm{d}, \mathrm{opt}}=10^{3.0} \mathrm{kN}$ for the stiff towers; $S I_{\min }^{(0)}=0.73$ and $c_{\mathrm{d}, \mathrm{opt}}=10^{2.8} \mathrm{kN}$ for the medium towers; $S I_{\min }^{(0)}=0.81$ and $c_{\mathrm{d}, \mathrm{opt}}=10^{2.5} \mathrm{kN}$ for the flexible towers; while for the three reaction towers together, one gets $\bar{V}_{\max }^{(\mathrm{T})}=19.0,13.9$ and 11.8 MN, respectively. A similar trend is observed for set 2 (see Figure 12b), although (as in the previous subsection), the optimal values $c_{\mathrm{d}, \text { opt }}$ for the NCR are higher than for the DLR.

Figures $12 \mathrm{a}$ and $12 \mathrm{~b}$ also show the effect of increasing the damper coefficient $c_{\mathrm{d}}$ on the total base shear, $V^{(0+\mathrm{T})}(t)=V^{(0)}(t)+V^{(\mathrm{T})}(t)$, experienced by existing building and reaction towers together; since in general the maxima for the individual sub-frames do not happen simultaneously, $\bar{V}_{\max }^{(0+\mathrm{T})}<\bar{V}_{\max }^{(0)}+\bar{V}_{\max }^{(\mathrm{T})}$. It has been found that the maximum total base shear at optimal damper constant for the three values of the stiffness ratio $\lambda$ is $43.7,46.4$ and $46.7 \mathrm{MN}$, respectively; these forces are slightly less than the corresponding ones attained for the unconnected configuration $(46.5,48.0$ and $46.9 \mathrm{MN}$ ), but show a consistent reduction (between 18 and $24 \%$ ) with respect to the case of rigidly connected towers $(58.1,60.3,57.4 \mathrm{MN}$, respectively).

Figures 13a and 13b display the profiles of the maximum displacements in the main building, $\bar{u}_{\max }^{(0)}$, for sets 1 and 2 , respectively. As expected, the larger $\lambda$, the less $\bar{u}_{\max }^{(0)}$ at all stories. The relatively small values of displacements and 


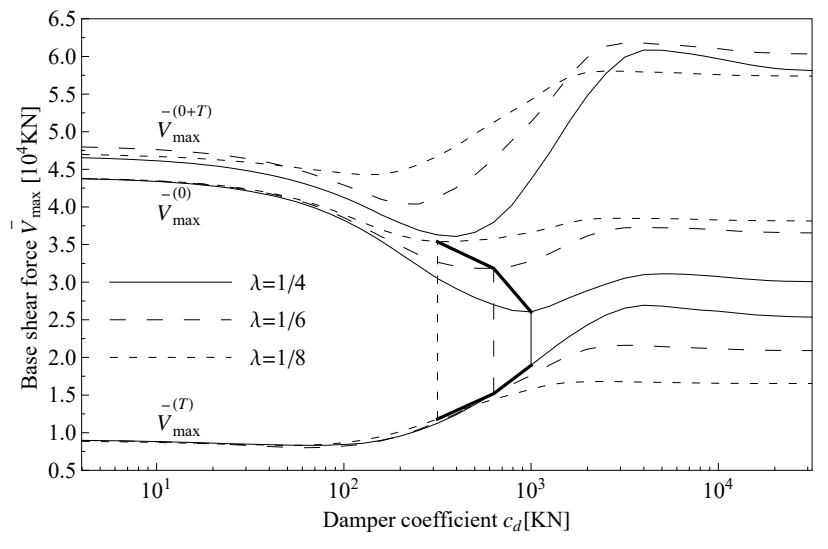

(a)

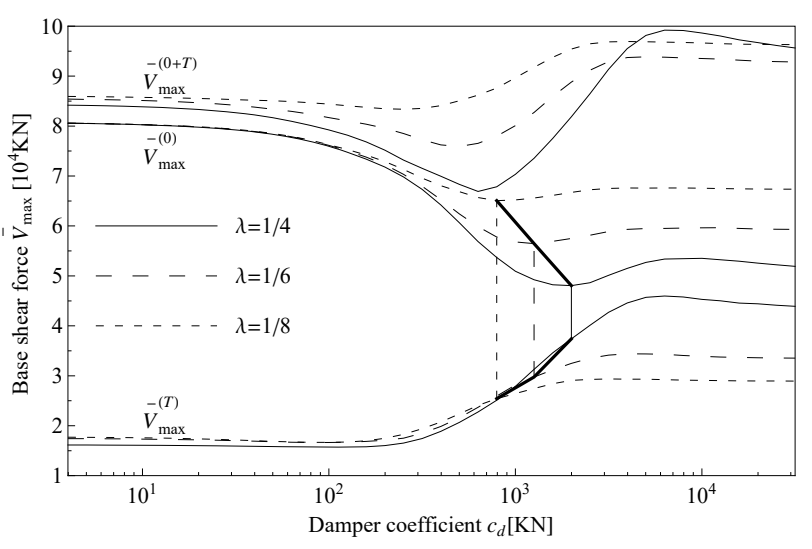

(b)

Figure 12. Maximum base shear vs. viscous damper coefficient for the velocity exponent $\alpha=0.15$, different stiffness ratios $1 / 8 \leq \lambda \leq 1 / 4$, seismic input in the $x$ direction and both DLR (a) and NCR (b) scenarios; the thick lines show the maximum base shear on the existing building and the three towers for the optimal viscous damper coefficients

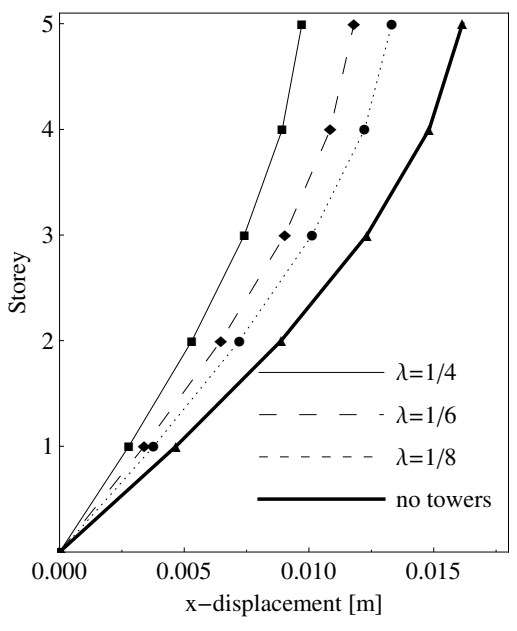

(a)

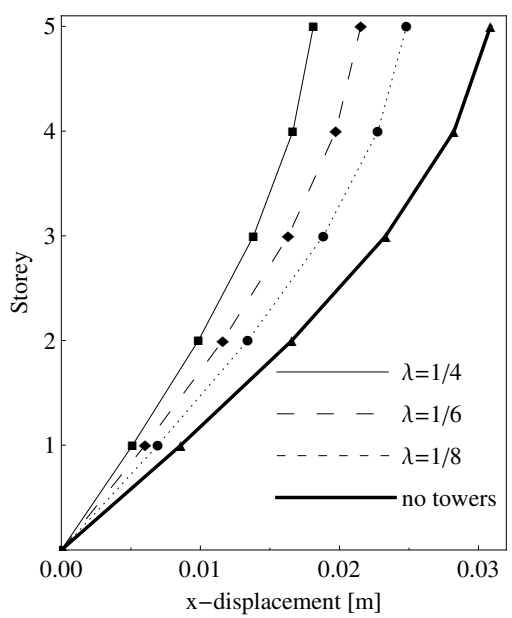

(b)

Figure 13. Profiles of maximum floor displacements for the unconnected (thick lines) and retrofitted building considering the seismic input in the $x$ direction, both DLR (a) and NCR (b) scenarios, the same velocity exponent $\alpha=0.15$ and different stiffness ratios $1 / 8 \leq \lambda \leq 1 / 4$

inter-story drifts calculated for the main building without reaction towers (solid lines) are due to the high stiffness of the masonry infill panels.

Furthermore, the improved performance of the damper-tower retrofitting strategy observed for stiffer towers appears to be in line with the findings of Trombetti and Silvestri [19], who have demonstrated for the case of linear devices the superiority of "mass-proportional" distribution of dampers, with respect to a "stiffness-proportional" distribution. The former case is achieved as limiting condition when $\lambda \rightarrow+\infty$, so that the damping forces become proportional to the velocities relative to the ground, while the latter case corresponds to the more common arrangement of the dampers installed between consecutive stories, thus with damping forces proportional to the relative velocities between two consecutive floors.

Lastly, the effects of $\lambda$ on the maximum force in the monitored damper at the top floor are analyzed in Figures 14a and $14 \mathrm{~b}$. Interestingly, $\bar{f}_{\mathrm{d} \text {,max }}$ is not sensitive to the stiffness ratio $\lambda$ (that is, only for very high values of $c_{\mathrm{d}}$ it is possible to observe some small differences in $\bar{f}_{\mathrm{d} \text {,max }}$ ). However, as expected, if the optimal damper constant is chosen, $c_{\mathrm{d}, \text { opt }}$, then the stiffer the tower, the higher the forces in the NLVD. 


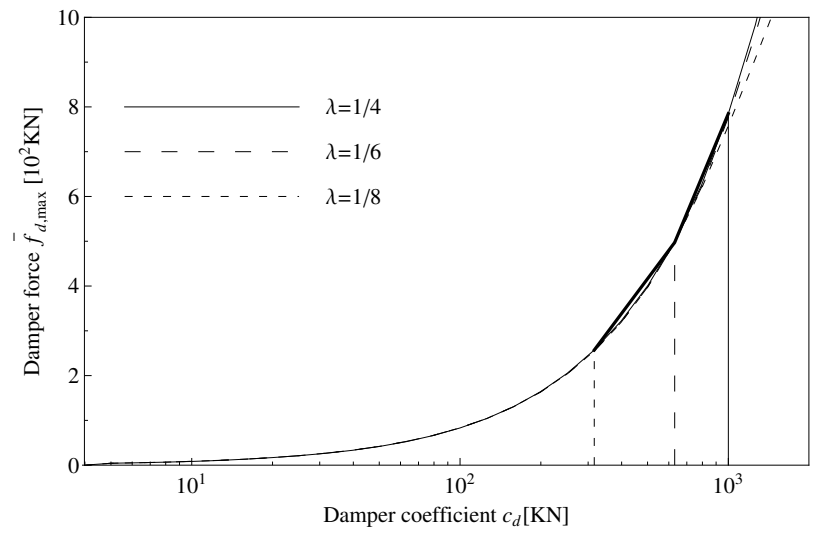

(a)

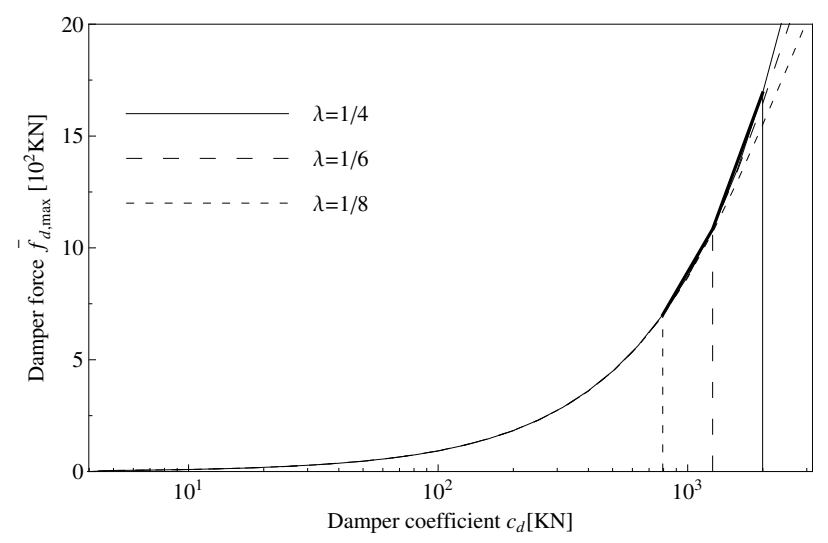

(b)

Figure 14. Variation with $c_{\mathrm{d}}$ of the maximum damper force between existing building and third reaction tower at the top floor for the velocity exponent $\alpha_{\mathrm{d}}=0.15$, different stiffness ratios $1 / 8 \leq \lambda \leq 1 / 4$, seismic input in the $x$ direction and both DLR (a) and NCR (b) scenarios; the thick lines show the maximum damper force for optimal damper

\subsection{Influence of the number of connected stories $n_{S}^{*}$}

An important design decision is whether NLVDs need to be installed at all floors (i.e. $n_{\mathrm{S}}^{*}=n_{\mathrm{S}}$ ), or a more costeffective retrofitting solution can be achieved with a reduced number of connected stories (i.e. $1 \leq n_{\mathrm{S}}^{*}<n_{\mathrm{S}}$ ). To investigate this aspect of the problem, six alternative configurations were considered, with $n_{\mathrm{S}}^{*}=2$ (three cases), $n_{\mathrm{S}}^{*}=3$ (two cases) and $n_{\mathrm{S}}^{*}=5$ (reference case). Once again, the numerical analyses assumed: $x$ as the direction of attack of the ground shaking $\left(\Theta_{\mathrm{g}}=0\right)$; velocity exponent $\alpha_{\mathrm{d}}=0.15$; stiffness ratio $\lambda=1 / 6$; while this time only the set 2 of accelerograms for the NCR were used.

For the case study under investigation, Figure 15 shows that dampers positioned at the lower stories can have a larger effect on the reduction of $\bar{V}_{\max }^{(0)}$ than dampers at the top stories, allowing to achieve for the main building almost the same value of $S I_{\min }^{(0)}$ as in the reference case with all stories connected to the reaction towers. However, as expected, the value of the optimal viscous damper coefficient increases when some stories are disconnected. Indeed, higher damper coefficients are required if only the lower stories are connected, similarly to what also reported by Aida et al. [14]. For instance, if only the first 3 stories are equipped with NLVDs, one gets $S I_{\min }^{(0)}=0.70$ and $c_{\mathrm{d} \text {,opt }}=10^{3.5} \mathrm{kN}$; very close values are obtained connecting only the first and the top story, while mounting NLVDs at all stories leads to the same $S I_{\min }^{(0)}=0.70$, while the viscous damper coefficient reduces to $c_{\mathrm{d}, \mathrm{opt}}=10^{3.1} \mathrm{kN}$.

In comparison, higher values of $S I_{\min }^{0)}$ are observed when only the top stories are connected, although the corresponding values of $c_{\mathrm{d} \text {,opt }}$ are closer to the optimal values for reference case; that is, the "valley" of $\bar{V}_{\max }^{(0)}$ in Figure 15 is less low, but the minimum is obtained almost for the same value of $c_{\mathrm{d} \text {,opt }}$. It should also be mentioned here that, for a given value of the damper constant $c_{\mathrm{d}}$, better performance are typically observed if the higher stories are connected, rather than the lower stories. Furthermore, connecting only the top stories rather than the bottom ones allows achieving a larger reduction in the shear force at those locations (see Figure 16), and thus a larger reduction in the inter-story drifts.

This trend of results can be explained by the different mechanisms through which the seismic behavior is optimized when only top or bottom stories are connected. In the first case, the prevalent mechanism is the increased amount of energy dissipation, which benefits from the larger amplitude of vibration at the top stories with respect to the bottom ones. In the second case, it seems that the prevalent mechanism is a reduction in the effective height of the vibrating structures, which in turn requires a higher level of restraint (and thus larger dampers) at the bottom stories. This trend also suggests that better performance can be achieved using simultaneously smaller dampers at the top and larger dampers at the bottom. Further investigations are required to better elucidate this aspect, which is beyond the scope of this paper.

In order to check the overall performance of the various configurations, the mean values of the maximum shear force at all stories are compared in Figure 16. Although the use NLVDs connected to the reaction towers is always beneficial, it appears that the number and position of the connected stories strongly affect the amount of shear force 


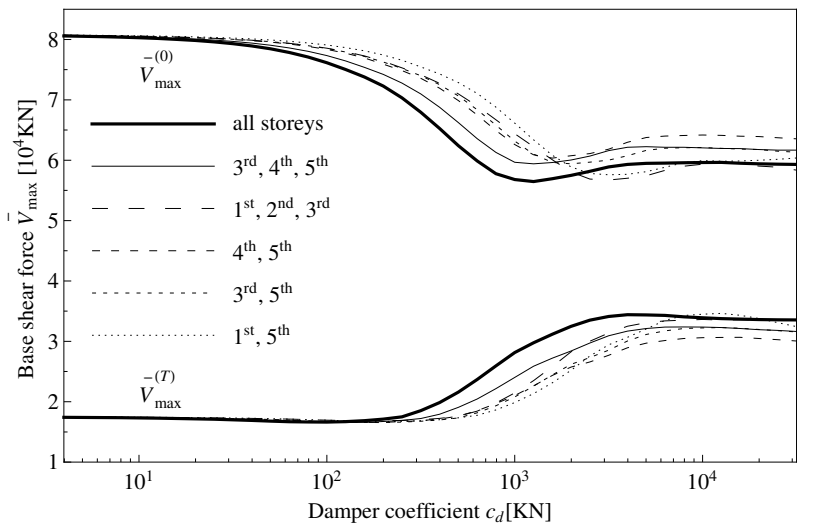

Figure 15. Maximum base shear vs. viscous damper coefficient for the velocity exponent $\alpha=0.15$, stiffness ratio $\lambda=1 / 6$, seismic input in the $x$ direction, NCR scenario and varying number and levels of connected stories

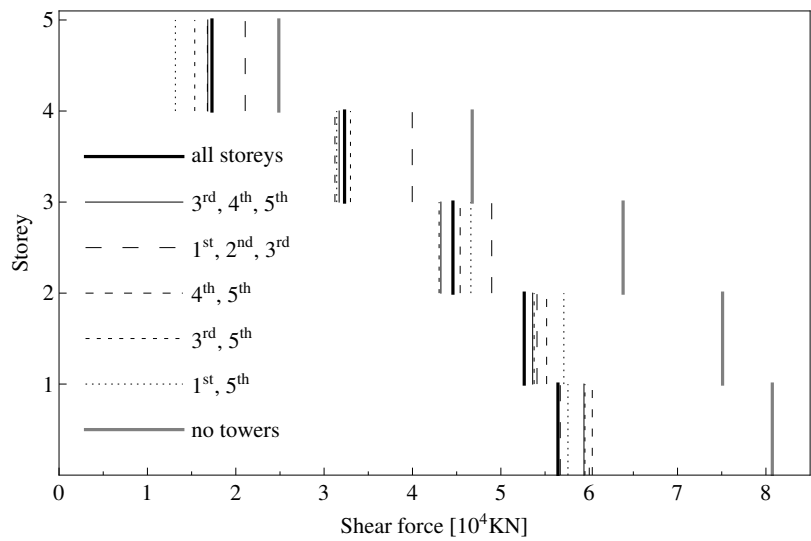

Figure 16. Profile of maximum shear forces of the unconnected (grey lines) and retrofitted building for the velocity exponent $\alpha=0.15$, stiffness ratio $\lambda=1 / 6$, seismic input in the $x$ direction, NCR scenario and varying number and levels of connected stories

reduction along the height of the main building, meaning that the key structural deficiencies in the existing building should be carefully considered when designing the retrofitting intervention.

Furthermore, existing building and reaction towers connected at all stories may not be the best solution if the overall cost of the intervention in taken into account. For instance, a reduced number of NLVDs, connected only at the 3rd and 5th floor, would provide the case-study building with almost the same level of earthquake protection as having all the floors connected to the reaction towers. Finally, a retrofitting strategy based on the introduction of NLVDs just at the lower stories may be not suitable to reduce the seismic demand at top stories, despite the good performance in terms of the base shear force.

\subsection{Lateral-torsional coupled vibrations: earthquake along the y direction}

When the earthquake loading is applied along the $y$ direction $\left(\Theta_{\mathrm{g}}=\pi / 2 \mathrm{rad}\right)$, trends of results similar to those for the $x$ direction are obtained in terms of performance indexes $S I^{(0)}$ and $D I^{(0)}$ (see Table II). In particular, Figure 17 shows $V_{\max }^{(0)}$ and $V_{\max }^{(\mathrm{T})}$ versus the viscous damper coefficient for the devices acting along the $y$ direction only. These curves have been obtained for the accelerograms of set 2 (NCR), different stiffness ratios $(1 / 8 \leq \lambda \leq 1 / 4)$ and keeping for the devices in the $x$ direction the same optimal values previously found for the seismic analysis in the $x$ direction. Interestingly, the same optimal coefficients have been obtained for the dampers along the $y$ direction, and also the shear indexes are similar: $S I_{\min }^{(0)}=0.59$ for $\lambda=1 / 4, S I_{\min }^{(0)}=0.72$ for $\lambda=1 / 6$ and $S I_{\min }^{(0)}=0.80$ for $\lambda=1 / 8$.

As expected, when the viscous damper coefficient increases, the reaction towers affect the torsional response of the main building by shifting its center of rigidity. Figure 18 reveals that the maximum top story rotation drops 


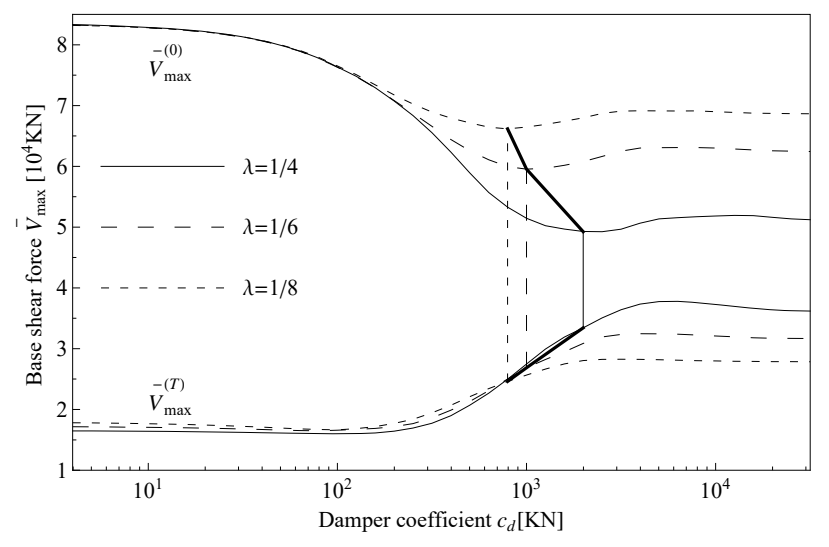

Figure 17. Maximum base shear vs. viscous damper coefficient in the $y$ direction for the velocity exponent $\alpha=0.15$, different stiffness ratios $1 / 8 \leq \lambda \leq 1 / 4$, seismic input in the $y$ direction and NCR scenario; the thick lines show the maximum base shear on the existing building and the three towers for the optimal viscous damper coefficients

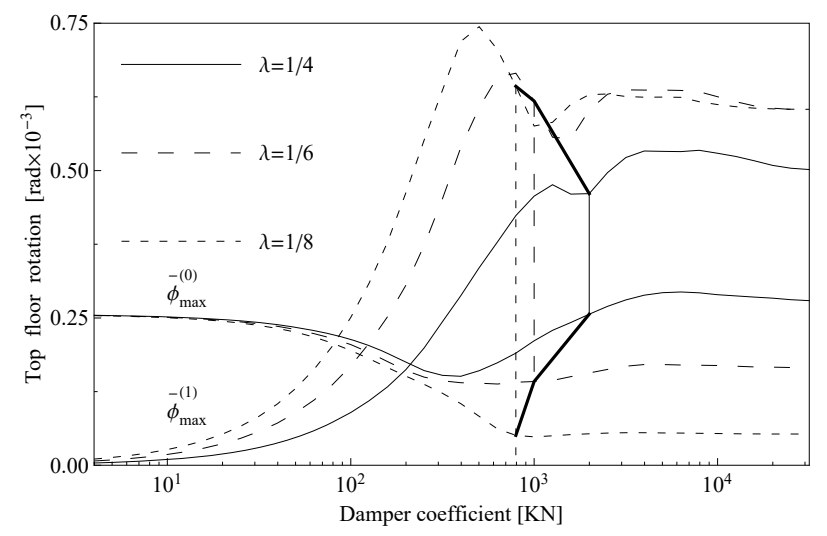

Figure 18. Maximum top floor rotations in the main building and in the first tower vs. viscous damper coefficient in the $y$ direction for the velocity exponent $\alpha=0.15$, different stiffness ratios $1 / 8 \leq \lambda \leq 1 / 4$, seismic input in the $y$ direction and NCR scenario; the thick lines show the maximum rotations for the optimal viscous damper coefficients

from $0.25 \times 10^{-3} \mathrm{rad}$ (unconnected towers) to a minimum of $0.048 \times 10^{-3} \mathrm{rad}$ when $\lambda=1 / 8$. If the tower stiffness increases further, the reduction is less, namely $0.14 \times 10^{-3} \mathrm{rad}$ for $\lambda=1 / 6$ and $0.15 \times 10^{-3}$ rad for $\lambda=1 / 4$. Furthermore, the lowest value for the maximum rotation is achieved for a viscous damper constant equal to $c_{\mathrm{d}, \mathrm{opt}}$ when $\lambda=1 / 8$, and smaller values when stiffness of the towers increases. These results demonstrate that, for the casestudy building, the flexible towers are capable of preventing torsional movements by shifting the center of rigidity of the main building to the right, closer to its center of mass. If stiffer towers are used, then the center of rigidity moves further to the right, exceeding the position of the center of mass, i.e. the eccentricity changes its sign. Based on the above observations, a better design could be achieved by optimizing the relative stiffness of the three reaction towers in the two directions, e.g. having a relatively stiffer tower 3 in the $y$ direction (i.e. $K_{y y}^{(3)} / K_{y y}^{(1)}>1.5$ ).

Similarly, the need to minimize the torsional effects may also suggest to reduce the viscous damper constant in the $y$ direction with respect to the optimal value found in the $x$ direction for the scenarios with $\lambda=1 / 6$ and $\lambda=1 / 4$. This is evident from Figure 19, which shows that in the latter two cases $c_{\mathrm{d} \text {,opt }}$ (identified with the thick solid line) produces significantly larger rotations with respect to the valley of the pertinent curves, which occurs at $c_{\mathrm{d}}=10^{2.8} \mathrm{kN}$ for $\lambda=1 / 4$ and $c_{\mathrm{d}}=10^{2.7} \mathrm{kN}$ for $\lambda=1 / 6$. Reducing $c_{\mathrm{d}}$ will result on a relatively small increment in the total base shear experienced by the main building (see Figure 17), which however will be more evenly distributed among its lateral resisting frames. 


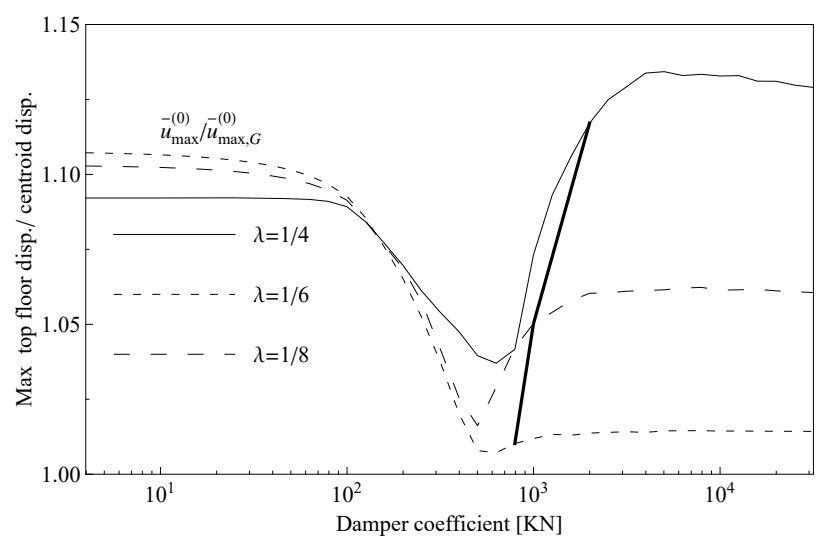

Figure 19. Ratio between maximum and centroid displacement at the top floor of the main building vs. viscous damper coefficient in the $y$ direction for the velocity exponent $\alpha=0.15$, different stiffness ratios $1 / 8 \leq \lambda \leq 1 / 4$, seismic input in

the $y$ direction and NCR scenario; the thick lines show the maximum rotations for the optimal viscous damper coefficients

\section{CONCLUSIONS}

The paper has demonstrated the technical viability of interventions of seismic retrofitting in which nonlinear viscous dampers (NLVDs) are not installed within the existing building (that might then require large relative displacements, often incompatible with the available ductility in its lateral resisting members, and might also be too complicated or too expensive considering various architectural and functional constraints), but between the primary structure of the existing building and a set of secondary structures, working as (relatively flexible) reaction towers.

An extensive set of numerical simulations have been carried out on a real case-study building (in excess of 60,000), potentially representative of a broad range of design situations, which supports the following general conclusions:

1. The combined effect of the additional stiffness provided by the reaction towers and the additional energy dissipation provided by the NLVDs allows reducing internal forces and inter-story drifts in the existing building (see Table II).

2. For a given stiffness ratio $\lambda$ between existing building and reaction towers, the degree of nonlinearity of the NLVDs (controlled by the velocity exponent $\alpha_{\mathrm{d}}$ ) has little or no effect on the reduction of the base shear in the existing building (see Figure 6); on the other hand, the higher degree of nonlinearity, the less the reaction forces in the viscous dampers (see Figure 7), and for this reason a lower value of the velocity exponent is advisable (e.g. $\left.\alpha_{\mathrm{d}}=0.15\right)$.

3. For given values of stiffness ratio $\lambda$ and velocity exponent $\alpha_{\mathrm{d}}$, the optimal viscous damper coefficient, $c_{\mathrm{d}, \mathrm{opt}}$, depends on the particular selection of the set of accelerograms defining the seismic input, but the actual reduction of the base shear in the existing building is less sensitive to them (see Figure 10); this is particularly important in the design stage, as the uncertainty in the characteristics of the seismic event marginally affects the expected efficiency of the retrofitting intervention.

4. The optimal viscous damper coefficient $c_{\mathrm{d}, \text { opt }}$ increases with the intensity of the seismic action (see Figure 10); considering the effects of the nonlinearity of the devices (see Figure 11), it is advisable to size them for the NCR (no-collapse requirement), i.e. the seismic event with a higher return period.

5. Stiffer reaction towers (i.e. higher values of $\lambda$ ) result in a larger reduction of the base shear in the existing building and higher forces in the reaction towers (see Figure 12), including their foundations; when designing the retrofitting intervention, thus, a trade-off should be sought between increased cost and improved seismic protection associated with stiffer reaction towers.

6. Connecting existing building and reaction towers at all stories guarantees the best performance of the retrofitting intervention; however, in monetary terms, a more efficient solution can be found installing the NLVDs at selected stories (see Figures 15 and 16) or possibly using different dampers at different stories.

7. The proposed retrofitting approach allows mitigating the negative effects that irregularities in plan can have on the dynamics of the existing building; in particular, stiffness of the reaction towers and viscous damper coefficients can be optimized to reduce the torsional movement in the main building (see Figures 17 to 19). 
Although derived from a single case-study, the above conclusions show that using NLVDs in conjunction with purposely built (or indeed already existing) structures can be an efficient way to achieve the seismic retrofitting of buildings with low ductility. As the amount of the resulting improvement will necessarily depend on the structural characteristics of the retrofitted building (e.g. fundamental period of vibration, number of stories, irregularities in plan and elevation, level of ductility, etcetera) and of the earthquake hazard (intensity, frequency content, etcetera), the numerical formulation established in Section 2 will therefore be used in future research to investigate a wider range of case studies, so to identify further trends and support a more detailed design strategy for this type of seismic retrofitting.

\section{REFERENCES}

[1] Italian Ministry of Infrastructure. Nuove NormeTecniche per le Costruzioni 29 2008. In Italian.

[2] Castellano MG, Colato GP, Infanti S. Use of viscous dampers and shock transmission units in the seismic protection of buildings. 13th World Conference on Earthquake Engineering, 2004.

[3] Lavan O, Levy R. Optimal design of supplemental viscous dampers for linear framed structures. Earthquake Engineering \& Structural Dynamics 2006; 35(3):337-356.

[4] Sorace S, Terenzi G. Seismic protection of frame structures by fluid viscous damped braces. Journal of Structural Engineering - ASCE 2008; 134(1):45-55.

[5] Cimellaro G, Roh H, De Stefano A. Spectral and fragility evaluations of retrofitted structures through strength reduction and enhanced damping. Earthquake Engineering and Engineering Vibration 2009; 8(1):115-125.

[6] Palmeri A, Muscolino G. A numerical method for the time-domain dynamic analysis of buildings equipped with viscoelastic dampers. Structural Control and Health Monitoring 2011; 18(5):519-539.

[7] Lavan O, Amir O. Simultaneous topology and sizing optimization of viscous dampers in seismic retrofitting of 3D irregular frame structures. Earthquake Engineering \& Structural Dynamics 2014; 43(9):1325-1342.

[8] Palmeri A. Passive control techniques for retrofitting of existing structures. Encyclopedia of Earthquake Engineering, Beer M, Kougioumtzoglou IA, Patelli E, Au SK (eds.). Springer, 2015; 1849-1871. ISBN: 9783642353437.

[9] Benavent-Climent A, Morillas L, Escolano-Margarit D. Seismic performance and damage evaluation of a reinforced concrete frame with hysteretic dampers through shake-table tests. Earthquake Engineering \& Structural Dynamics 2014; 43(15):2399-2417.

[10] Impollonia N, Avellino G, Trombetta P, Palmeri A. Seismic retrofitting of buildings by fluid viscous dampers connected to adjacent reaction towers. Safety, Reliability, Risk and Life-Cycle Performance of Structures and Infrastructures Proceedings of the 11th International Conference on Structural Safety and Reliability, ICOSSAR 2013, 2013; 1743-1750.

[11] Xu Y, He Q, Ko J. Dynamic response of damper-connected adjacent buildings under earthquake excitation. Engineering Structures 1999; 21(2):135-148.

[12] Zhang W, Xu Y. Vibration analysis of two buildings linked by Maxwell model-defined fluid dampers. Journal of Sound and Vibration 2000; 233(5):775-796.

[13] Zhang W, Xu Y. Dynamic characteristics and seismic response of adjacent buildings linked by discrete dampers. Earthquake Engineering \& Structural Dynamics 1999; 28(10):1163-1185.

[14] Aida T, Aso T, Takeshita K, Takiuchi T, Fujii T. Improvement of the structure damping performance by interconnection. Journal of Sound and Vibration 2001; 242(2):333-353.

[15] Ni Y, Ko J, Ying Z. Random seismic response analysis of adjacent buildings coupled with non-linear hysteretic dampers. Journal of Sound and Vibration 2001; 246(3):403-417.

[16] Patel C, Jangid R. Seismic response of adjacent structures connected with Maxwell dampers. Asian Journal of Civil Engineering 2010; 11(5):585-603.

[17] Yang Z, Xu Y, Lu X. Experimental seismic study of adjacent buildings with fluid dampers. Journal of Structural Engineering - ASCE 2003; 129(2):197-205.

[18] Bigdeli K, Hare W, Tesfamariam S. Configuration optimization of dampers for adjacent buildings under seismic excitations. Engineering Optimization 2012; 44(12):1491-1509.

[19] Trombetti T, Silvestri S. Added viscous dampers in shear-type structures: The effectiveness of mass proportional damping. Journal of Earthquake Engineering 2004; 8(2):275-313.

[20] Lavan O. On the efficiency of viscous dampers in reducing various seismic responses of wall structures. Earthquake Engineering \& Structural Dynamics 2012; 41(12):1673-1692.

[21] Lavan O, Abecassis D. Seismic behavior and design of wall-EDD-frame systems. Frontiers in Built Environment $2015 ; 1$.

[22] Martinez-Rodrigo M, Romero M. An optimum retrofit strategy for moment resisting frames with nonlinear viscous dampers for seismic applications. Engineering Structures 2003; 25(7):913-925.

[23] Dall'Asta A, Tubaldi E, Ragni L. Influence of the nonlinear behavior of viscous dampers on the seismic demand hazard of building frames. Earthquake Engineering \& Structural Dynamics 2016; 45(1):149-169. 
[24] Zhu H, Ge D, Huang X. Optimum connecting dampers to reduce the seismic responses of parallel structures. Journal of Sound and Vibration 2011; 330(9):1931-1949.

[25] Tubaldi E. Dynamic behavior of adjacent buildings connected by linear viscous/viscoelastic dampers. Structural Control and Health Monitoring 2015; 22(8):1086-1102.

[26] Tubaldi E, Barbato M, Ghazizadeh S. A probabilistic performance-based risk assessment approach for seismic pounding with efficient application to linear systems. Structural Safety 2012; 36-37:14-22.

[27] Tubaldi E, Barbato M, Dall'Asta A. Performance-based seismic risk assessment for buildings equipped with linear and nonlinear viscous dampers. Engineering Structures 2014; 78:90-99.

[28] Yang Z, Lam E. Dynamic responses of two buildings connected by viscoelastic dampers under bidirectional earthquake excitations. Earthquake Engineering \& Structural Dynamics 2014; 13(1):137-150.

[29] Roia D, Gara F, Speranza E, Gioiella L, Dezi L. Operational modal analysis on a r.c. building for the evaluation of the dynamic changes due to retrofitting. IEEE Workshop on Environmental, Energy and Structural Monitoring Systems, EESMS 2015, 2015.

[30] Passoni C, Belleri A, Marini A, Riva P. Existing structures connected with dampers: state of the art and future developments. 2nd European Conference on Earthquake Engineering and Seismology, 2014.

[31] Clough R, Penzien J. Dynamics of Structures. 2 (revised) edn., Computers and Structures, Inc, 2010.

[32] Kasinos S, Palmeri A, Lombardo M. Seismic response of combined primary-secondary structures with the componentmode synthesis method. Proceedings of the 15th International Conference on Civil, Structural and Environmental Engineering Computing, CC2015, 2015.

[33] Computers and Structures, Inc. SAP2000 v18 2015. https : / /www. csiamerica. com/products / sap2000.

[34] Palmeri A, Barone G, Khetawat A. Passive control of building structures using double-skin facades as vibration absorbers. 15th International Conference on Civil, Structural and Environmental Engineering Computing, 2015.

[35] Pipitone G, Barone G, Palmeri A. Optimal design of double-skin façades as vibration absorbers. Structural Control and Health Monitoring (In press); .

[36] European Committee for Standardization. Eurocode 8 - Design of Structures for Earthquake Resistance. Part 1: General Rules, Seismic Actions and Rules for Buildings 2004.

[37] Encyclopedia of Mathematics. Runge-Kutta method 2012. https://www. encyclopediaofmath. org/index. php/Runge-Kutta_method.

[38] Iervolino I, Galasso C, Cosenza E. Rexel: Computer aided record selection for code-based seismic structural analysis. Bulletin of Earthquake Engineering 2010; 8(2):339-362.

[39] Ambraseys N, Smit P, Sigbjornsson R, Suhadolc P, Margaris B. Internet-Site for European Strong-Motion Data. European Commission, Directorate-General for Research 2002. http://www.isesd.hi.is/ESD_Local/frameset. htm. 